\title{
DISK AND ENVELOPE STRUCTURE IN CLASS 0 PROTOSTARS. II. HIGH-RESOLUTION MILLIMETER MAPPING OF THE SERPENS SAMPLE
}

\author{
Melissa L. Enoch ${ }^{1}$, Stuartt Corder ${ }^{2}$, Gaspard Duchêne ${ }^{1,3}$, Douglas C. Bock ${ }^{4}$, Alberto D. Bolatto ${ }^{5}$, \\ Thomas L. Culverhouse ${ }^{6}$, Woojin Kwon ${ }^{7}$, James W. Lamb ${ }^{6}$, Erik M. Leitch ${ }^{6,8}$, Daniel P. Marrone ${ }^{8,11}$, \\ Stephen J. Muchovej ${ }^{6}$, Laura M. Pérez ${ }^{9}$, Stephen L. Scott ${ }^{6}$, Peter J. Teuben ${ }^{5}$, Melvyn C. H. Wright ${ }^{1}$, \\ AND B. Ashley ZaUderer ${ }^{5,10}$ \\ ${ }^{1}$ Department of Astronomy, University of California at Berkeley, 601 Campbell Hall, Berkeley, CA 94720, USA \\ ${ }^{2}$ NRAO/ALMA-JAO, Av. Apoquindo 3650, Piso 18, Las Condes, Santiago, Chile \\ ${ }^{3}$ UJF-Grenoble 1/CNRS-INSU, Institut de Planétologie et dAstrophysique de Grenoble (IPAG) UMR 5274, Grenoble, F-38041, France \\ ${ }^{4}$ Combined Array for Research in Millimeter-wave Astronomy, Big Pine, CA 93513, USA \\ ${ }^{5}$ Department of Astronomy and Laboratory for Millimeter-wave Astronomy, University of Maryland, College Park, MD 20742, USA \\ ${ }^{6}$ Owens Valley Radio Observatory, California Institute of Technology, Big Pine, CA 93513, USA \\ ${ }^{7}$ Department of Astronomy, University of Illinois, Urbana, IL 61801, USA \\ 8 Department of Astronomy and Astrophysics, University of Chicago, 5640 S. Ellis Ave. Chicago, IL 60637, USA \\ ${ }^{9}$ Department of Astronomy, California Institute of Technology, 1200 East California Blvd, Pasadena, CA 91125, USA \\ ${ }^{10}$ Harvard-Smithsonian Center for Astrophysics, 60 Garden Street, Cambridge, MA 02138, USA \\ Received 2010 November 19; accepted 2011 June 1; published 2011 August 4
}

\begin{abstract}
We present high-resolution CARMA $230 \mathrm{GHz}$ continuum imaging of nine deeply embedded protostars in the Serpens Molecular Cloud, including six of the nine known Class 0 protostars in Serpens. This work is part of a program to characterize disk and envelope properties for a complete sample of Class 0 protostars in nearby low-mass star-forming regions. Here, we present CARMA maps and visibility amplitudes as a function of $u v$-distance for the Serpens sample. Observations are made in the B, C, D, and E antenna configurations, with B configuration observations utilizing the CARMA Paired Antenna Calibration System. Combining data from multiple configurations provides excellent $u v$-coverage (4-500 k $\lambda$ ), allowing us to trace spatial scales from $10^{2}$ to $10^{4} \mathrm{AU}$. We find evidence for compact disk components in all of the observed Class 0 protostars, suggesting that disks form at very early times $(t<0.2 \mathrm{Myr})$ in Serpens. We make a first estimate of disk masses using the flux at $50 \mathrm{k} \lambda$, where the contribution from the envelope should be negligible, assuming an unresolved disk. The resulting disk masses range from $0.04 M_{\odot}$ to $1.7 M_{\odot}$, with a mean of approximately $0.2 M_{\odot}$. Our high-resolution maps are also sensitive to binary or multiple sources with separations $\gtrsim 250 \mathrm{AU}$, but significant evidence of multiplicity on scales $<2000 \mathrm{AU}$ is seen in only one source.
\end{abstract}

Key words: ISM: individual objects (Serpens) - stars: formation - submillimeter: ISM - techniques: interferometric

Online-only material: color figure

\section{INTRODUCTION}

Circum-stellar and circum-protostellar disks are an important component of the star formation process, transferring mass from the surrounding envelope to the growing central protostar. While disk formation is a natural result of collapse in a rotating core, it is still not known how soon after protostellar formation the disk appears, or how massive it is at early times. Simple analytical theories of star formation suggest that centrifugally supported disks should start out quite small (radius $<10 \mathrm{AU}$ ), and consequently with very low mass, and grow with time (Terebey et al. 1984). More recent simulations of self-gravitating and viscous disks indicate that relatively massive disks can form at very early times (e.g., Vorobyov 2009). Magnetically supported disks can also be much larger (radii up to $1000 \mathrm{AU}$; Galli \& Shu 1993), and thus more massive at early times.

To understand the formation and growth of disks, and their relationship to the accretion process, it is critical to observe and characterize disks in embedded sources, where most of the mass of the growing star is being accreted. Directly observing disks in the embedded phases is much more difficult than in more evolved pre-main-sequence stars, as they are hidden within the dense, extincting protostellar envelopes.

\footnotetext{
${ }^{11}$ Hubble Fellow.
}

Embedded protostars are defined here as protostars with substantial $\left(>0.1 M_{\odot}\right)$ envelope masses remaining. We will also refer to young sources by class, where the bolometric temperature $T_{\mathrm{bol}}{ }^{12}$ (Myers \& Ladd 1993; Chen et al. 1995) is used to divide sources into Class $0\left(T_{\text {bol }} \leqslant 70 \mathrm{~K}\right)$, Class I $\left(70 \mathrm{~K}<T_{\text {bol }} \leqslant 650 \mathrm{~K}\right)$, and Class II $\left(650 \mathrm{~K}<T_{\text {bol }} \leqslant 2800 \mathrm{~K}\right)$. Here, we assume that these classes correspond to an evolutionary sequence (e.g., Robitaille et al. 2006; André 1994): in Class 0 the protostar has accreted less than half its final mass $\left(M_{*}<M_{\text {env }}\right)$, in Class I more than half of the mass has been accreted $\left(M_{*}>M_{\mathrm{env}}\right)$, and in Class II the envelope has dispersed, leaving only a circum-stellar disk.

In the last two decades a number of studies have focused on detecting disks in embedded protostars (e.g., Chandler et al. 1995; Brown et al. 2000; Looney et al. 2000; Harvey et al. 2003; Jørgensen et al. 2005a; Eisner et al. 2005; Andrews \& Williams 2007). These studies have found that disks are well established in Class I, with typical masses of $0.01-0.5 M_{\odot}$. Although circumprotostellar disks have been detected in a number of Class 0 sources, most detailed studies of Class 0 sources have been

\footnotetext{
12 The bolometric temperature is the temperature of a blackbody with the same mean frequency as the observed spectral energy distribution

$\left(T_{\text {bol }}=1.25 \times 10^{-11}\langle v\rangle \mathrm{K}\right.$; Myers \& Ladd 1993) and is a better measure of the evolutionary state than the infrared spectral index (Enoch et al. 2009b).
} 
limited to the brightest or most well-known objects due to instrumental limitations, a lack of complete target samples, and the difficulty of separating disk and envelope emission in such young sources.

Jørgensen et al. (2007, 2009) have recently completed a large Submillimeter Array (SMA) survey of 10 Class 0 and 10 Class I sources, finding similar disk masses in Class 0 and Class I. Although Jørgensen et al. (2009) see no clear evolutionary signature in disk flux or mass, they do see an increase in the ratio of disk mass to envelope mass with evolutionary stage. The SMA study still relies on well-known sources from different regions, however, rather than a complete or well-defined sample. A study characterizing "typical" Class 0 disks, in a sample that is complete down to some well-defined luminosity or envelope mass limit, is still needed.

We have recently begun a program to characterize disks and envelopes in a complete sample of Class 0 protostars in nearby low-mass star-forming regions. Combining Combined Array for Research in Millimeter-Wave Astronomy (CARMA) $230 \mathrm{GHz}$ continuum imaging and Spitzer Infrared Spectrograph (IRS) mid-infrared (MIR) with radiative transfer modeling of this sample will help to address several fundamental questions about the structure and evolution of the youngest protostars. (1) How soon after the initial collapse of the parent core does a circumprotostellar disk form? (2) What fraction of the total circumprotostellar mass resides in the disk, and does this fraction vary with time? (3) Are large "holes" in the inner envelope (e.g., Jørgensen et al. 2005b) common at early times?

Millimeter maps and MIR spectra provide complementary approaches to these questions. The amount of flux escaping at $\lambda \lesssim 50 \mu \mathrm{m}$ from embedded protostars is sensitive to the opacity close to the protostar and thus the envelope structure (e.g., Jørgensen et al. 2005b). While the MIR flux is insensitive to disk properties, high-resolution millimeter continuum mapping directly detects emission from dust grains in the disk. Millimeter observations with good $u v$-coverage and calibration, combined with radiative transfer models, can separate the disk from the envelope and constrain the disk mass and size.

Our ultimate goal is to characterize the disk mass, size, and inner envelope structure of typical low-mass Class 0 protostars, and to quantify any trends with evolutionary indicators. In Enoch et al. (2009a), hereafter Paper I, we presented results for Serpens FIRS 1, a well-known Class 0 source, as a pilot for the full program. We found that disks can be quite massive at very early times; radiative transfer modeling (RADMC; Dullemond \& Dominik 2004) compared to CARMA visibilities and spectral information indicated that FIRS 1 has a disk with mass $M_{\text {disk }}=1.0 M_{\odot}$ and radius $R_{\text {disk }}=300 \mathrm{AU}$, making up approximately $13 \%$ of the total circum-protostellar mass in a source that is about $10^{5}$ years old.

Here, we present high-resolution millimeter imaging of nine embedded protostars in the Serpens Molecular Cloud, including six of the nine known Class 0 sources in the cloud. The CARMA $^{13}$ is an ideal instrument for studying the disk properties of deeply embedded protostars. With 15 antennas operating at $\lambda=1 \mathrm{~mm}$, CARMA provides the excellent $u v$-coverage required to separate disk emission from envelope emission in young protostars.

In Section 2 we describe the Serpens sample, and in Section 3 outline the CARMA observations and data reduction. CARMA maps and visibilities are presented in Section 4, followed by an

\footnotetext{
13 http://www.mmarray.org/
}

initial estimate of disk masses from the visibility amplitude at large $u v$-distances in Section 5. Individual sources are discussed in Section 6. We comment on the observed multiplicity fraction in our sample in Section 7.

\section{SERPENS SAMPLE}

Recent large surveys of nearby molecular clouds at midinfrared and (sub)millimeter wavelengths have made it possible to define complete samples of Class 0 protostars based on luminosity or envelope mass limits (e.g., Hatchell et al. 2007; Jørgensen et al. 2008; Dunham et al. 2008; Enoch et al. 2009b; Evans et al. 2009). Our study is based on the complete sample of 39 Class 0 protostars in the Serpens, Perseus, and Ophiuchus molecular clouds, identified by Enoch et al. (2009b), identified by comparing large-scale Spitzer Infrared Array Camera (IRAC) and Multi-Band Imaging Photometer for Spitzer (MIPS) with Bolocam (Glenn et al. 2003) $1.1 \mathrm{~mm}$ continuum surveys of the three clouds. Here we focus on the Serpens cloud, which contains 34 embedded protostars, 9 Class 0 sources and 25 Class I, and is complete to envelope masses $M_{\text {env }} \gtrsim 0.25 M_{\odot}$ and internal luminosities $L_{\text {int }} \gtrsim 0.05 L_{\odot}$.

The Serpens molecular cloud is an active star-forming region with high extinction and a high density of young stellar objects (YSOs). It has been studied extensively at near-infrared, farinfrared, submillimeter, and millimeter wavelengths (e.g., Eiroa \& Casali 1992; Hurt \& Barsony 1996; Larsson et al. 2000; Davis et al. 1999; Casali et al. 1993; Testi \& Sargent 1998). We adopt a distance of $d=415 \pm 25$ pc based recent Very Long Baseline Array (VLBA) trigonometric parallax measurements of a YSO in Serpens (Dzib et al. 2010). Note that this is significantly larger than the $d=260 \mathrm{pc}$ that we have assumed in our previous work (Paper I; Enoch et al. 2007, 2009b). To compare to our previous disk and envelope masses in Serpens, masses given here should be decreased by a factor of 2.5 and physical sizes by 1.6. Mass ratios will remain unchanged.

Targets are listed in Table 1, including the "Ser-emb\#" name from Enoch et al. (2009b), Spitzer position, bolometric luminosity $\left(L_{\text {bol }}\right)$, bolometric temperature $\left(T_{\text {bol }}\right)$, and total envelope mass $\left(M_{\text {env }}\right)$. Our goal here is to image all known Class 0 protostars in Serpens with high resolution and high fidelity with CARMA. Enoch et al. (2009b) found nine Class 0 protostars in Serpens. Three of the nine known Class 0 source were not included in the $230 \mathrm{GHz}$ observations, however. These three sources were either not detected or only marginally detected, in preliminary $110 \mathrm{GHz}$ lower-resolution CARMA maps (see the Appendix).

All undetected sources are in confused regions, with multiple Spitzer sources associated with a single Bolocam $1.1 \mathrm{~mm}$ core; at least one source in each core is detected and included here. The undetected sources do not have particularly low luminosity or low envelope mass (see Table 1), so excluding them does not bias the survey by luminosity or circum-protostellar mass. In addition to six Class 0 targets, we include three Class I sources that are near the Class 0/I cutoff.

Two sources in our sample (Ser-emb 4 and emb 6) were observed as part of the Hogerheijde et al. (1999) interferometric study of four deeply embedded protostars in Serpens, but of the two only Ser-emb 6 was detected. The other two Hogerheijde et al. (1999) sources were classified as Class I by Spitzer.

As described in Enoch et al. (2009b), $L_{\text {bol }}$ and $T_{\text {bol }}$ are calculated from the spectral energy distribution (SED) from $1.25 \mu \mathrm{m}$ to $1100 \mu \mathrm{m}$. Broadband infrared data for our targets are taken from the "From Molecular Cloud Cores to Planet 
Table 1

Serpens Targets

\begin{tabular}{|c|c|c|c|c|c|c|c|c|}
\hline ID & $\begin{array}{l}\text { c2d Name/Position } \\
(\text { SSTc2dJ...) }\end{array}$ & $\begin{array}{l}T_{\text {bol }} \\
(\mathrm{K})\end{array}$ & $\begin{array}{c}L_{\text {bol }} \\
\left(L_{\odot}\right)\end{array}$ & $\begin{array}{l}M_{\text {env }} \\
\left(M_{\odot}\right)\end{array}$ & Class & Bolocam ID & $230 \mathrm{GHz}$ Map? & $\begin{array}{l}\text { Other } \\
\text { Names }\end{array}$ \\
\hline Ser-emb 1 & J182909.24+003132.3 & 39 & 4.1 & 3.1 & Class 0 & Bolo 15 & $\mathrm{Y}$ & \\
\hline Ser-emb 2 & $\mathrm{~J} 182952.44+003611.7$ & 42 & 2.5 & 1.3 & Class 0 & Bolo 24 & $\mathrm{~N}$ & \\
\hline Ser-emb 3 & $\mathrm{~J} 182854.84+002952.5$ & 51 & 6.6 & 3.8 & Class 0 & Bolo 8 & $\mathrm{~N}$ & \\
\hline Ser-emb 4 & $\mathrm{~J} 183000.72+011301.4$ & 54 & 3.1 & 6.6 & Class 0 & Bolo 28 & $\mathrm{Y}$ & \\
\hline Ser-emb 5 & J182854.84+001832.6 & 56 & 0.4 & 0.5 & Class 0 & Bolo 7 & $\mathrm{Y}$ & \\
\hline Ser-emb 7 & J182854.12+002929.9 & 58 & 7.9 & 4.3 & Class 0 & Bolo 8 & $\mathrm{Y}$ & \\
\hline Ser-emb 8 & $\mathrm{~J} 182948.12+011644.9$ & 58 & $5.4^{\mathrm{a}}$ & 9.4 & Class 0 & Bolo 22 & $\mathrm{Y}$ & S68N \\
\hline Ser-emb 9 & $\mathrm{~J} 182855.92+002944.7$ & 66 & 2.5 & 6.6 & Class 0 & Bolo 8 & $\mathrm{~N}$ & \\
\hline Ser-emb 11 & J182906.72+003034.3 & 77 & 4.8 & 3.6 & Class I & Bolo 14 & $\mathrm{Y}$ & \\
\hline Ser-emb 15 & J182954.24+003601.3 & 100 & 0.4 & 1.3 & Class I & Bolo 24 & $\mathrm{Y}$ & \\
\hline Ser-emb 17 & J182906.36+003043.2 & 120 & 3.8 & 3.6 & Class I & Bolo 14 & $\mathrm{Y}$ & \\
\hline
\end{tabular}

Notes. Position is the Spitzer source position. $T_{\mathrm{bol}}$ and $L_{\mathrm{bol}}$ are calculated from the broadband SED from $1.25 \mu \mathrm{m}$ to $1100 \mu \mathrm{m}$ (2MASS, IRAC, MIPS, Bolocam) and $M_{\text {env }}$ from the total $1.1 \mathrm{~mm}$ flux, as described in Enoch et al. (2009b). Note that both $L_{\text {bol }}$ and $M_{\text {env }}$ are larger by a factor of 2.5 than those listed in Enoch et al. (2009b) due to the larger cloud distance assumed here. "Bolocam ID" denotes the Bolocam 1.1 mm core with which the source is associated; note that more than one embedded protostar is often associated with a given Bolocam core, due to the $31^{\prime \prime}$ Bolocam resolution. "230 GHz map?" indicates if the source is included in the $230 \mathrm{GHz}$ study.

a Source is saturated at $70 \mu \mathrm{m}$, so $L_{\text {bol }}$ is likely an underestimate.

Forming Disks" Spitzer Legacy program ("Cores to Disks" or c2d; Evans et al. 2003), which imaged $1 \mathrm{deg}^{2}$ in the cloud with IRAC and MIPS (Harvey et al. 2006, 2007). The same region was mapped at $\lambda=1.1 \mathrm{~mm}$ with the Bolocam bolometer array at the Caltech Submillimeter Observatory (Enoch et al. 2007). Together with public Two Micron All Sky Survey (2MASS) catalogs, these data provide wavelength coverage at $1.25,1.65$, $2.17 \mu \mathrm{m}$ (2MASS), 3.6, 4.5, 5.8, $8.0 \mu \mathrm{m}$ (IRAC), 24, 70, $160 \mu \mathrm{m}$ (MIPS), and $1100 \mu \mathrm{m}$ (Bolocam).

The envelope mass $M_{\text {env }}$ is calculated from the total $\lambda=$ $1.1 \mathrm{~mm}$ single dish flux, assuming that the envelope is optically thin at $1.1 \mathrm{~mm}$, a dust opacity of $\kappa_{1 \mathrm{~mm}}=0.0114 \mathrm{~cm}^{2} \mathrm{~g}^{-1}$ (Ossenkopf \& Henning 1994), a dust temperature of $T_{D}=15 \mathrm{~K}$, and $d=415$ pc. Here, we treat the envelope mass as the total circum-protostellar mass, as it may include some flux contribution from a disk component.

Figure 1 gives an overview of the Serpens environment with Spitzer $24 \mu \mathrm{m}$ (gray scale) and Bolocam $1.1 \mathrm{~mm}$ continuum (contours) maps. The locations of our embedded protostar targets are indicated. Most are associated with the Serpens Main cluster to the north or Cluster B (Djupvik et al. 2006; Harvey et al. 2006) to the south, but a few are more isolated. Three color Spitzer images $(8,24,70 \mu \mathrm{m})$ of the nine targets imaged with CARMA are shown in Figure 2, with Bolocam $1.1 \mathrm{~mm}$ contours as in Figure 1. SEDs of each source from $1.25 \mu \mathrm{m}$ to $1100 \mu \mathrm{m}$ can be found in Enoch et al. (2009b).

\section{OBSERVATIONS AND DATA REDUCTION}

Continuum observations at $v=230 \mathrm{GHz}(\lambda=1.3 \mathrm{~mm})$ were completed with CARMA, a 23 element interferometer consisting of nine $6.1 \mathrm{~m}$, six $10.4 \mathrm{~m}$, and eight $3.5 \mathrm{~m}$ antennas. The $6.1 \mathrm{~m}$ and $10.4 \mathrm{~m}$ antennas were used to obtain $230 \mathrm{GHz}$ continuum observations in the B (100-1000 m baselines), C (30-350 m), D (11-150 m), and E (8-66 m) antenna configurations between 2007 October and 2010 January. Three to four sources were observed in each track; although the $u v$-coverage is not identical for each target, every source was observed in each configuration, and at least $\pm 3 \mathrm{hr}$ around transit. Integration times varied based on the expected flux from single dish observations.
Three correlator bands were configured for continuum observations with $468.75 \mathrm{MHz}$ bandwidth, for a total bandwidth of $2.82 \mathrm{GHz}$ (two sidebands per band). A bright quasar (1751+096) was observed approximately every 15-20 minutes, to be used for complex gain calibration. For B configuration tracks, a secondary calibrator (1830+063) was also observed every 15 minutes. Absolute flux calibration was accomplished using 5 minute observations of Uranus, Neptune, or MWC 349. The overall calibration uncertainty is approximately $\pm 20 \%$, from the reproducibility of the phase calibrator flux on nearby days. A passband calibrator, typically 3C454.3, was observed for 15 minutes during each set of observations, and either optical (Corder et al. 2010) or radio pointing was performed every one to three hours.

Data from different array configurations were combined to provide excellent $u v$-coverage from 4 to $500 \mathrm{k} \lambda$, providing sensitivity to physical scales from 170 AU to $2 \times 10^{4}$ AU. Small seven-point mosaics were made in the compact configurations (D and E) in order to mitigate spatial filtering by the interferometer. Mosaicing provides better sensitivity to the spatially extended protostellar envelopes.

Observations in the most extended B configuration utilized the CARMA Paired Antenna Calibration System (CPACS) to correct for phase variations on sub-minute timescales (Pérez et al. 2010). For the longest B configuration baselines, C-PACS pairs $6.1 \mathrm{~m}$ and $10.4 \mathrm{~m}$ antennas (science array) with $3.5 \mathrm{~m}$ antennas (reference array) that operate at $\lambda=1 \mathrm{~cm}$. The reference array continually monitors the atmospheric phase using a strong point source close to the science target (here $1751+096$, also the gain calibrator, which is $\sim 13$ deg away), while the science array slews between the science targets and gain calibrator. The delay measured by the reference array is later used to correct the science data for atmospheric variations (see Pérez et al. 2010 for details). The $3.5 \mathrm{~m}$ antennas were tuned to $30 \mathrm{GHz}$, with a 14-band wideband correlator providing $7 \mathrm{GHz}$ of bandwidth.

Calibration and imaging are accomplished with the MIRIAD data reduction package (Sault et al. 1995). Calibration of each track consists of applying line-length calibration, passband correction, phase correction on 15-30 minute intervals, amplitude calibration on 60 minute intervals, and absolute flux calibration. 


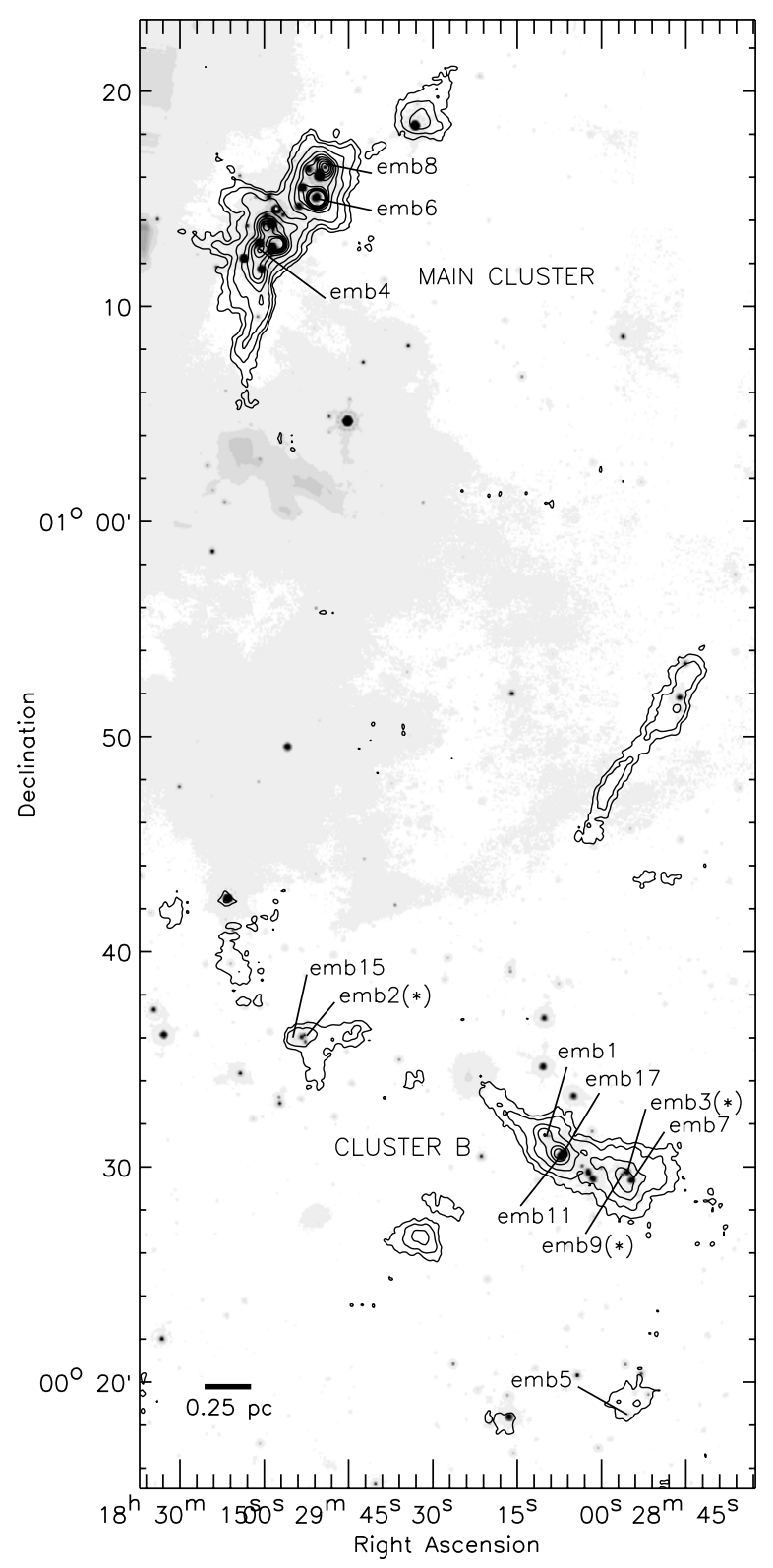

Figure 1. Overview of the Serpens Main cluster and Cluster B region, with a finding chart for embedded protostars observed with CARMA. The Spitzer $24 \mu \mathrm{m}$ map from c2d is shown in gray scale, with Bolocam $1.1 \mathrm{~mm}$ continuum contours overlaid (contours are 50,100, 200, 400,600, .., $1400 \mathrm{mJy} \mathrm{beam}^{-1}$ ). Although the Serpens cloud, as defined by the $A_{V}=6$ contour, extends beyond the region shown, all embedded protostars are found within this area. "emb\#" designations are from Enoch et al. (2009b), and sources marked with a "(*)" are Class 0 protostars not included in the $230 \mathrm{GHz}$ study (see Sections 2 and the Appendix).

For B configuration data, delays are calculated from the $30 \mathrm{GHz}$ observations of $1751+096$ and applied to each paired baseline on $4 \mathrm{~s}$ intervals after passband calibration. The $230 \mathrm{GHz}$ gain calibrator observations are then used to apply longerinterval (15 minute) phase corrections, to remove slowly varying instrumental delay differences between the reference and science arrays. C-PACS corrections usually work best for close ( $<6 \mathrm{deg}$ ) atmospheric calibrators (B. A. Zauderer et al. 2011, in preparation); given that $1751+096$ is 13 deg from our science targets, we only apply C-PACS calibration for tracks where it significantly improves phase coherence on the secondary calibrator (1830+063, $5 \mathrm{deg}$ from the science targets). When ap- plied, C-PACS calibration improves the signal to noise of the peak flux of $1830+063$ by a factor of $1.5-6$.

Images are formed by inverting the calibrated visibilities using multi-frequency synthesis with natural weighting to optimize signal to noise, cleaned with a Steer CLEAN algorithm (Steer et al. 1984), and restored with a Gaussian fit to the synthesized beam. Beam sizes for the combined maps (including all configurations) are approximately $1^{\prime \prime}$, corresponding to a physical resolution of $415 \mathrm{AU}$.

We also calculate the azimuthally averaged visibility amplitude as a function of $u v$-distance. Due to mosaicing in the compact arrays, visibilities must be combined over multiple pointing centers and are calculated in three steps. The visibilities are first modified in phase to account for any offset between the source position and the pointing position. The visibilities are then divided by the primary beam response at the location of the peak of the flux in the various pointings. Finally, we plot the weighted mean of the visibilities over the different pointing positions. With this method, very extended components or bright multiple sources can introduce errors in the visibility amplitudes. However, if the emission is very compact relative to the $30^{\prime \prime}$ primary beam, the error introduced by this scaling of the visibilities from the offset pointings is minimal.

\section{MAPS AND VISIBILITIES}

The resulting $230 \mathrm{GHz}$ maps are shown in Figure 3. In addition to maps including all data, two maps including only $u v$-distances $<50 \mathrm{~K} \lambda$ and $>50 \mathrm{~K} \lambda$ are shown, to better illustrate any extended and compact emission, respectively.

The $230 \mathrm{GHz}$ peak flux, total flux, and deconvolved size of each detected source, determined from a Gaussian fit (MIRIAD's imfit) to the combined map, are given in Table 2. The rms noise level in the central region, excluding sources, is also listed (ranging between 0.9 and $8 \mathrm{mJy}^{\text {beam }}{ }^{-1}$ ), along with the synthesized beam size or resolution (typically $\sim 1^{\prime \prime}$ or $415 \mathrm{AU}$ ). Multiple entries are given when more than one source is detected in the CARMA map.

Plots of the visibility amplitude versus $u v$-distance for each embedded protostar are shown in Figure 4. Extended envelope structures, as expected for embedded Class 0 protostars, are visible as an amplitude peak at $u v$-distances $<20 \mathrm{k} \lambda$ in the visibility plots (Figure 4). Only Ser-emb 15 does not show evidence for a strong peak at $u v$-distance $<20 \mathrm{~K} \lambda$. The peak visibility amplitude is equivalent to the total $230 \mathrm{GHz}$ flux of the source. ${ }^{14}$ The interferometer does filter out flux at $u v$-distances less than $4 \mathrm{k} \lambda$ (physical scales $>2 \times 10^{4} \mathrm{AU}$ ), corresponding to the separation of the closest antenna pairs, so the total flux observed by CARMA will often be less than the total single dish flux.

A point source, such as an unresolved disk, will appear as a constant amplitude in the visibility plot, while a resolved disk will contribute an additional wide Gaussian component to the visibilities. Although the most common interpretation of a compact visibility component is a disk, without dynamical information or independent constraints on the envelope structure, compact emission could also be attributed to a power-law envelope (Looney et al. 2003) or non-axisymmetric envelope structure (Looney et al. 2007). With these caveats in mind, here we interpret compact emission as evidence of a circum-protostellar disk.

\footnotetext{
14 Note that this can be larger than the total flux listed in Table 2 because a Gaussian fit does not always capture the full extent of an extended source.
} 

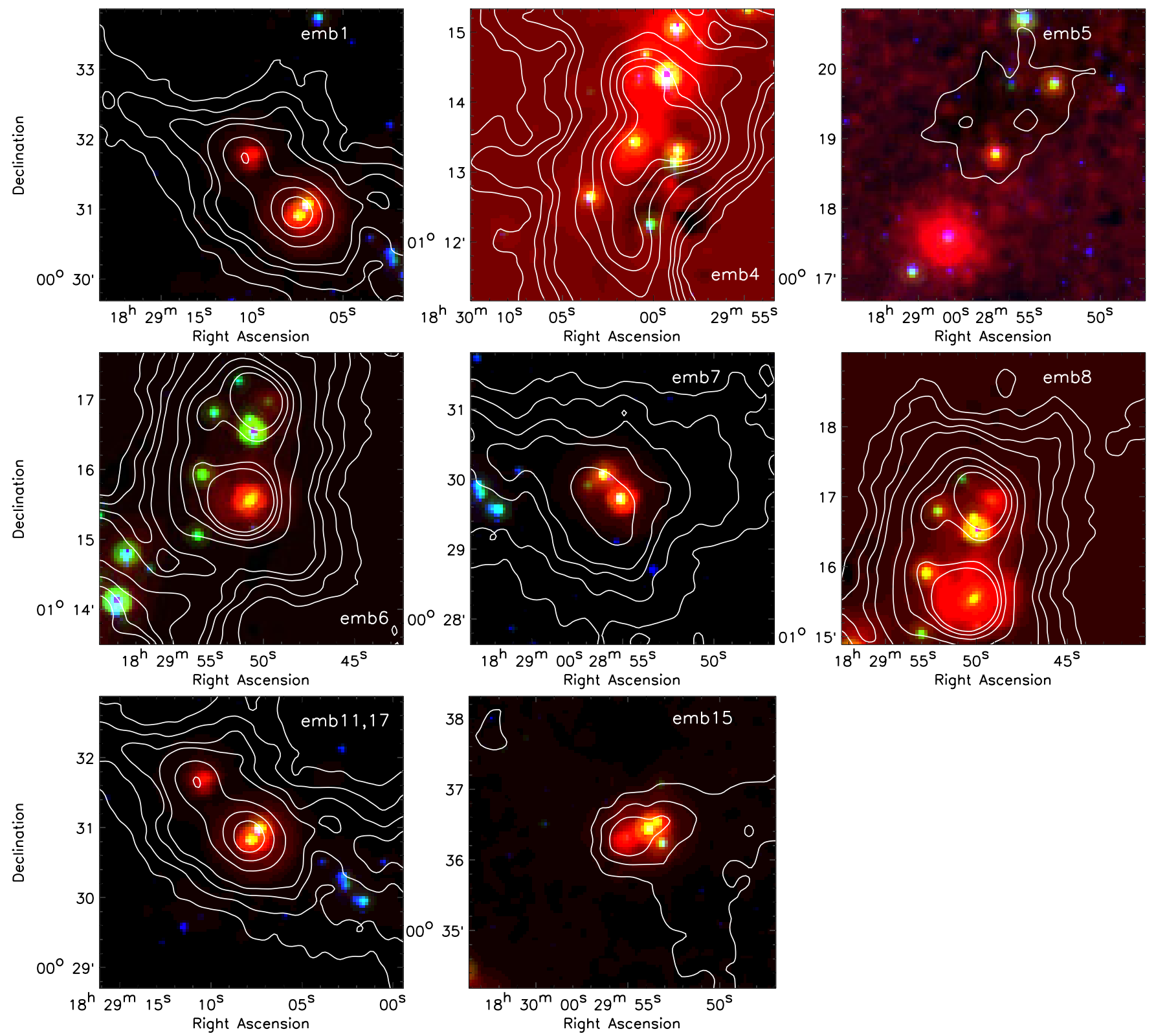

Figure 2. Spitzer three color $(8 \mu \mathrm{m}, 24 \mu \mathrm{m}, 70 \mu \mathrm{m})$ images of the nine embedded protostars imaged here with CARMA. Bolocam $1.1 \mathrm{~mm}$ continuum contours are shown at 50,100, 150, 200, 400,600, 800, 1000 mJy beam $^{-1}$. Each target is centered; Ser-emb 11 and emb 17 are $<15^{\prime \prime}$ apart and are shown in the same panel. (A color version of this figure is available in the online journal.)

Table 2

CARMA $230 \mathrm{GHz}$ Source Properties

\begin{tabular}{lcccccc}
\hline \hline ID & 230 GHz Position & $\begin{array}{c}\text { Peak Flux } \\
\left(\mathrm{mJy}^{-1}\right)\end{array}$ & $\begin{array}{c}\text { Total Flux } \\
(\mathrm{mJy})\end{array}$ & $\begin{array}{c}\text { rms } \\
\left(\mathrm{mJy}^{-1}\right)\end{array}$ & $\begin{array}{c}\text { Beam } \\
(\operatorname{arcsec})\end{array}$ & $\begin{array}{c}\text { Deconvolved Size } \\
(\operatorname{arcsec})\end{array}$ \\
\hline Ser-emb 1 & $18: 29: 09.09+00: 31: 30.9$ & 94.7 & 215 & 3.0 & $1.2 \times 1.1$ & $1.5 \times 1.1$ \\
Ser-emb 4 (N) & $18: 30: 00.30+01: 12: 59.4$ & 6.3 & 245 & 1.5 & $1.2 \times 1.1$ & $8.2 \times 6.3$ \\
Ser-emb 4 (E) & $18: 30: 00.70+01: 12: 56.2$ & 6.1 & 30.3 & 1.5 & $1.2 \times 1.1$ & $2.6 \times 2.1$ \\
Ser-emb 4 (S) & $18: 30: 00.62+01: 12: 49.5$ & 5.6 & 130 & 1.5 & $1.2 \times 1.1$ & $7.2 \times 4.3$ \\
Ser-emb 5 & $18: 28: 54.91+00: 18: 32.4$ & 6.1 & 14.3 & 0.8 & $1.3 \times 1.2$ & $1.6 \times 1.2$ \\
Ser-emb 6 & $18: 29: 49.79+01: 15: 20.4$ & 423 & 1390 & 6.7 & $0.9 \times 0.9$ & $1.6 \times 1.2$ \\
Ser-emb 7 & $18: 28: 54.04+00: 29: 29.7$ & 9.7 & 281 & 1.9 & $1.0 \times 1.0$ & $5.9 \times 4.6$ \\
Ser-emb 8 & $18: 29: 48.07+01: 16: 43.7$ & 40.1 & 3970 & 4.3 & $0.7 \times 0.6$ & $7.0 \times 5.9$ \\
Ser-emb 11 (W) & $18: 29: 06.61+00: 30: 34.0$ & 25.6 & 107 & 2.8 & $1.0 \times 1.0$ & $1.9 \times 1.6$ \\
Ser-emb 11 (E) & $18: 29: 06.75+00: 30: 34.4$ & 13.3 & 55.2 & 2.8 & $1.0 \times 1.0$ & $1.8 \times 1.6$ \\
Ser-emb 15 & $18: 29: 54.30+00: 36: 00.8$ & 41.5 & 77.5 & 1.7 & $1.0 \times 1.0$ & $1.1 \times 0.8$ \\
Ser-emb 17 & $18: 29: 06.20+00: 30: 43.1$ & 42.2 & 109 & 2.8 & $1.0 \times 1.0$ & $1.3 \times 1.2$ \\
\hline
\end{tabular}

Notes. Peak fluxes, total fluxes, and source sizes are determined by a Gaussian fit in the combined $230 \mathrm{GHz}$ maps. The $1 \sigma$ rms is calculated in the central $15^{\prime \prime}$ of each map, excluding bright sources. An angular size of $1^{\prime \prime}$ corresponds to a physical size of 415 AU. 

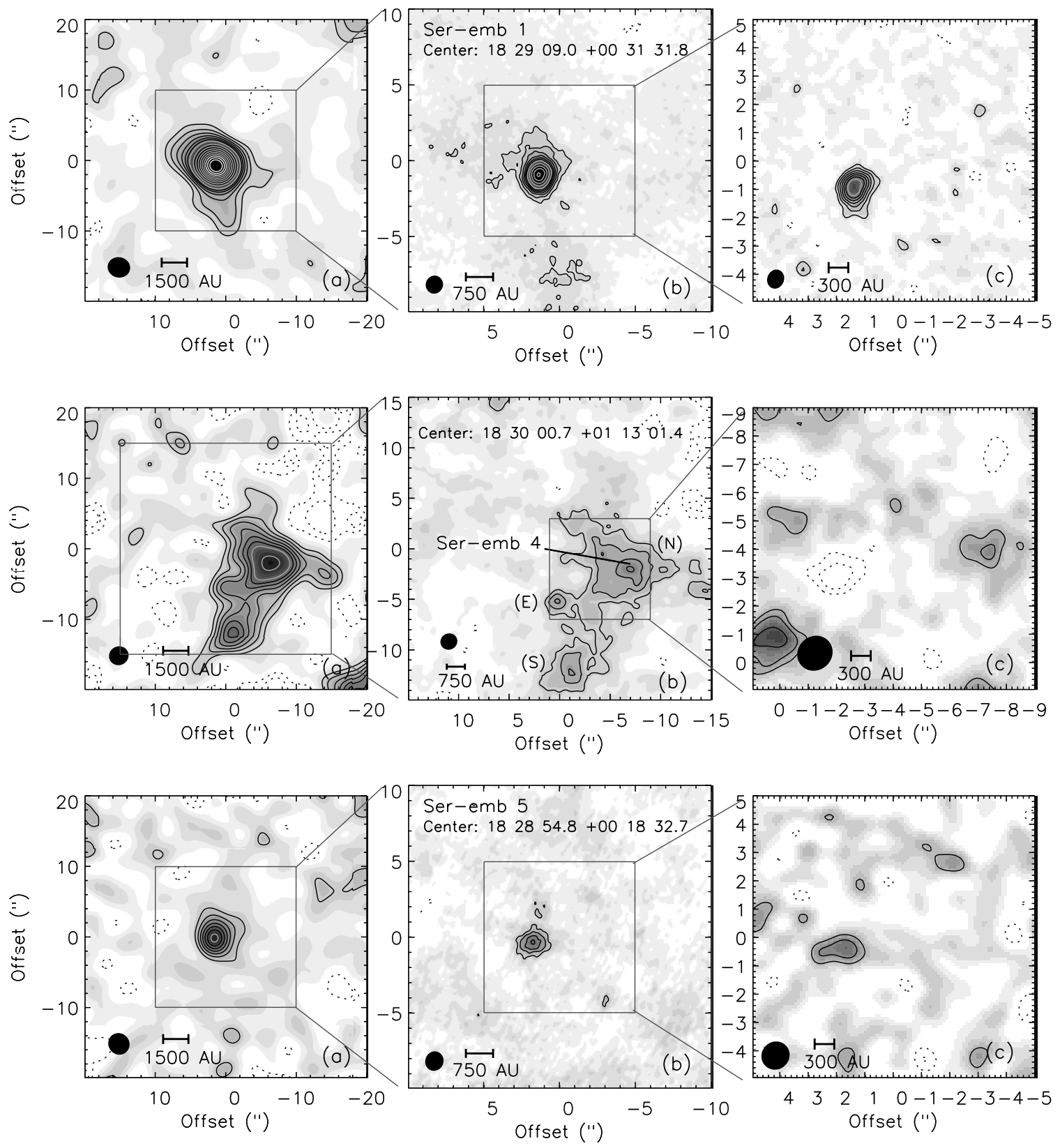

(a)

Figure 3. CARMA $230 \mathrm{GHz}$ images of Serpens deeply embedded protostars. The combined maps, shown in panel (b), include data from B, C, D, and E configurations, with seven-point mosaics in D and E. Large-scale and small-scale emission maps are shown in panels (a) and (c), respectively; maps in panel (a) are made including only $u v$-distances $<50 \mathrm{~K} \lambda$, and maps in panel (c) with $u v$-distances $>50 \mathrm{~K} \lambda$ (with the exception of Ser-emb 4 , which includes $u v$-distances $>30 \mathrm{~K} \lambda$ due to low signal to noise on long baselines). Contours are shown at $3 \sigma, 5 \sigma, \ldots, 15 \sigma, 20 \sigma, \ldots, 60 \sigma, 80 \sigma, \ldots, 140 \sigma$, where $\sigma$ is given in Table 2 , with lighter contours starting at $15 \sigma$ and negative contours indicated by dotted lines. Synthesized beam sizes (approximately 1" or 400 AU for combined maps) are indicated by solid ellipses. Offsets from the pointing centers are likely due to errors in the Spitzer positions, which are determined by the shorter wavelength data where outflows can cause extended or offset emission.

We can see immediate evidence for a compact disk component, as non-zero amplitudes at $u v$-distances $>50 \mathrm{k} \lambda$, in most sources (e.g., Ser-emb 4, emb 7, emb 11, emb 17, emb 15). Other sources have significant, but non-constant, flux for $u v$-distance $50-100 \mathrm{k} \lambda$, which may be due to a partially resolved disk (e.g., Ser-emb 1, emb 6, emb 8).

\section{ESTIMATED DISK MASSES}

Accurately measuring disk masses requires comparing the $230 \mathrm{GHz}$ visibilities and spectral information with detailed ra- diative transfer models, as in Paper I. Radiative transfer models such as RADMC (Dullemond \& Dominik 2004) simultaneously model the envelope and the disk, taking into account any envelope component at small scales, as well as a possibly resolved disk. In addition, radiative transfer models self-consistently solve for the temperature as a function of position. Work to constrain the disk and envelope structure of these sources with grids of radiative transfer models is currently ongoing (M. L. Enoch et al. 2011, in preparation).

For now, we make a first estimate of the disk mass using the $230 \mathrm{GHz}$ flux at a $u v$-distance of $50 \mathrm{~K} \lambda$. An unresolved 

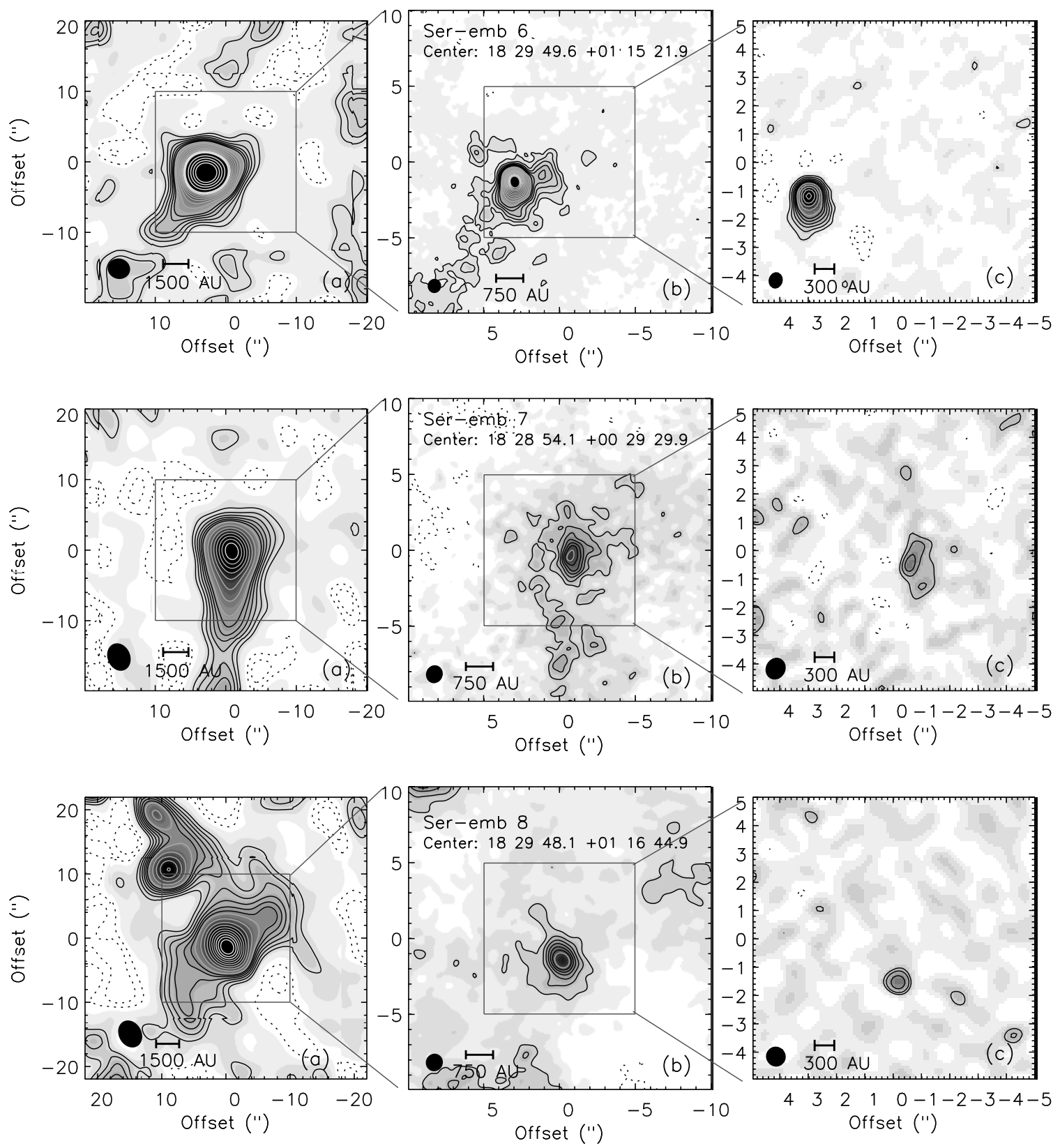

(b)

Figure 3. (Continued)

disk will have a constant flux independent of $u v$-distance, while the resolved envelope flux falls off quickly with $u v$-distance. Radiative transfer models of interferometric observations of a number of embedded protostars have indicated that protostellar envelopes contribute little emission at baselines longer than $50 \mathrm{~K} \lambda$ (e.g., Keene \& Masson 1990; Harvey et al. 2003; Jørgensen et al. 2005b). Using similar models to separate the disk and envelope in Ser-emb 6, we find that the envelope flux for $u v$-distances $>30 \mathrm{~K} \lambda$ is nearly zero (see Figure 6 of Paper I), contributing $<2 \%$ of the total flux at $50 \mathrm{~K} \lambda$.

The choice of $50 \mathrm{~K} \lambda$, as was adopted by Jørgensen et al. (2009), is a good compromise between minimizing the envelope contribution and maximizing the signal to noise of the disk, which falls off with increasing baseline length (due to decreased $u v$ coverage).
To calculate the mass, we assume that the disk is optically thin at $v=230 \mathrm{GHz}$ :

$$
M_{\text {disk }}=\frac{d^{2} S_{50 \mathrm{k} \lambda}}{B_{1.3 \mathrm{~mm}}\left(T_{D}\right) \kappa_{1.3 \mathrm{~mm}}},
$$

where $S_{50 \mathrm{k} \lambda}$ is the flux at $50 \mathrm{~K} \lambda, d=415 \mathrm{pc}$ is the cloud distance, and $B_{1.3 \mathrm{~mm}}$ is the Planck function for a dust temperature of $T_{D}=30 \mathrm{~K}$ (e.g., Visser et al. 2009; Jørgensen et al. 2009). We assume that the dust composition is similar in the disk and envelope, taking the dust opacity per gram of gas at $\lambda=1.3 \mathrm{~mm}$, $\kappa_{1.3 \mathrm{~mm}}=0.009 \mathrm{~cm}^{2} \mathrm{~g}^{-1}$, from Table 1 Column 6 of Ossenkopf \& Henning (1994), for dust grains with thin ice mantles.

Here, we assume that there is a negligible contribution by the envelope to the flux at $50 \mathrm{~K} \lambda$. If approximately $30 \%$ of the 

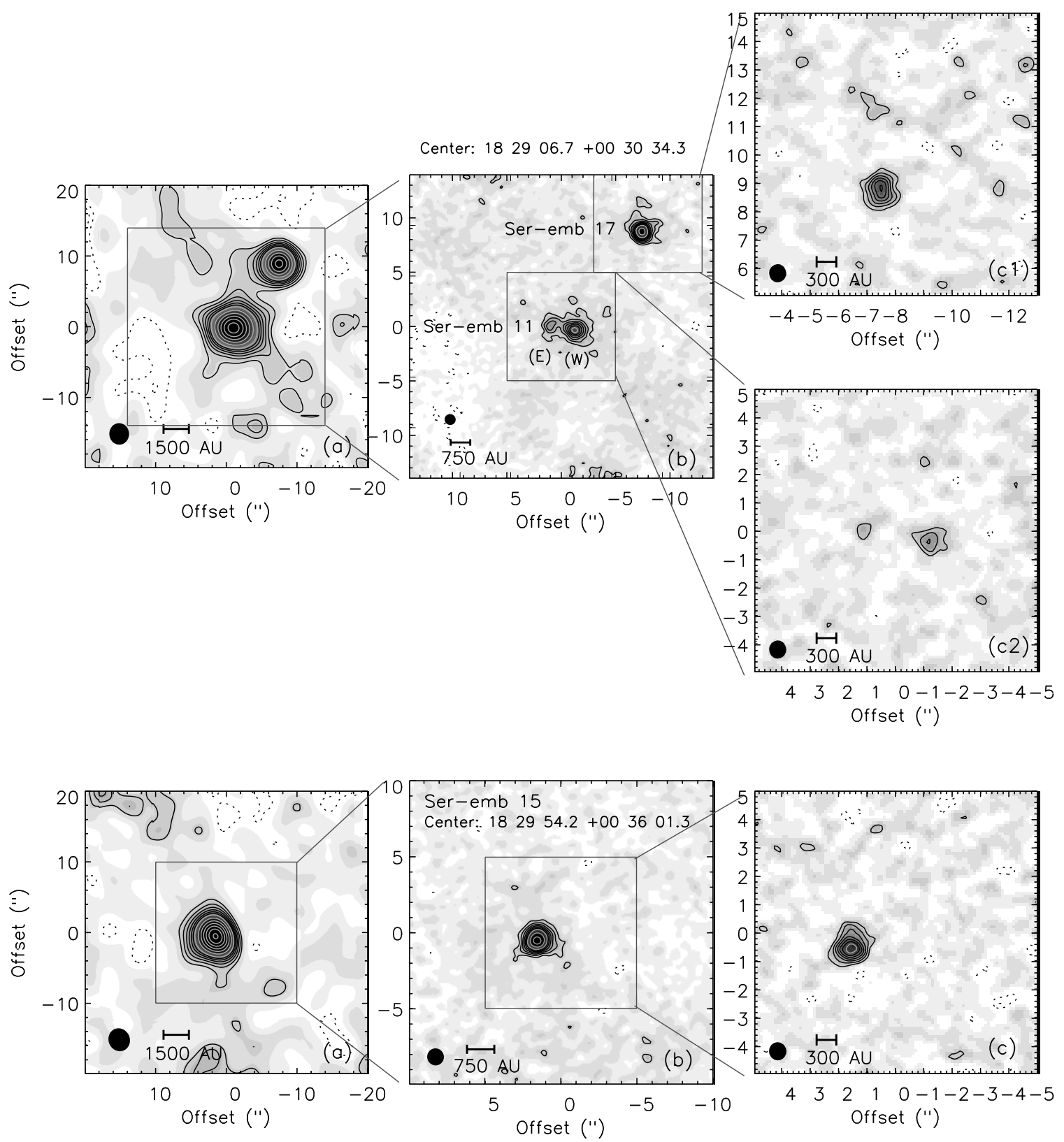

(c)

Figure 3. (Continued)

flux at $50 \mathrm{~K} \lambda$ can be contributed to the envelope, as found by Jørgensen et al. (2009) based on radiative transfer models of $8000 \mathrm{AU}$ envelopes, disk masses could decrease by $30 \%$.

On the other hand, if any of the disks are partially resolved (i.e., have radii larger than $170 \mathrm{AU}$ ), the flux at $50 \mathrm{~K} \lambda$ will underestimate the disk mass. For Ser-emb 6, the flux at $50 \mathrm{~K} \lambda$ suggests a mass that is $30 \%$ smaller than the mass from detailed radiative transfer modeling (Enoch et al. 2009a). Furthermore, if any part of the disk is optically thick, or the outer regions of the disk have a dust temperature well below $30 \mathrm{~K}$, the true mass of the disk could be considerably higher than our estimates. We therefore assign uncertainties to our estimated disk masses of at least $\pm 50 \%$.

Estimated disk masses are given in Table 3, and are plotted as a function of bolometric temperature, a measure of the evolutionary state, in Figure 5. We do not see a trend with evolutionary state in the narrow $T_{\text {bol }}$ range observed. The mean disk mass in our nine-source sample is $0.33 M_{\odot}$.

We must also account for the three Class 0 sources not included here due to non-detections in preliminary $110 \mathrm{GHz}$ maps. If Ser-emb 2, emb 3, or emb 9 had a compact disk with $M_{\text {disk }} \gtrsim 0.02 M_{\odot}$ they would have been detected at $3 \mathrm{~mm}$ (see the Appendix), so we assume that these sources do not have significant disks. Thus, we find evidence for circumprotostellar disks in six of the nine Class 0 sources in Serpens. If we include these non-detections, we find a mean disk mass $\left\langle M_{\text {disk }}\right\rangle=0.2 M_{\odot}$.

The disk to envelope mass ratio $\left(M_{\text {disk }} / M_{\text {env }}\right)$ is also shown versus $T_{\text {bol }}$ in Figure 5, where $M_{\text {env }}$ is from Table 2. Disk masses are between $1 \%$ and $10 \%$ as large as envelope masses in our 

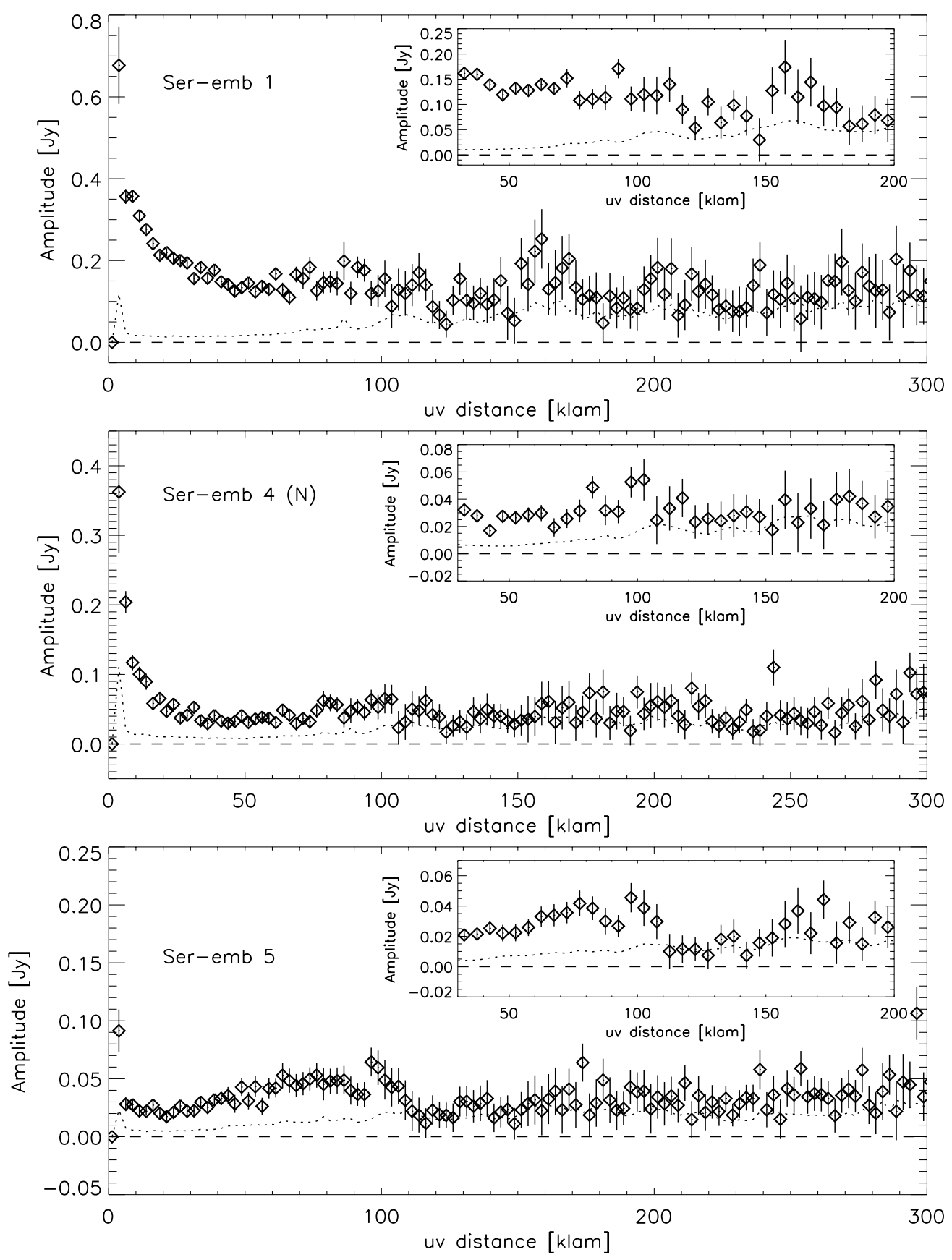

(a)

Figure 4. Azimuthally averaged visibility amplitudes as a function of $u v$-distance for the Serpens embedded protostars. The value expected for zero signal, or bias, is shown for reference (dotted line). Averaged visibilities are vector averaged at the measured source position (Table 2), and not the pointing center. Error bars reflect the variable $u v$-coverage, and atmospheric decorrelation on the longest baselines $(>100$ or $>200 \mathrm{~K} \lambda$ depending on source flux); they do not include the $\sim 20 \%$ absolute calibration uncertainty. Visibilities are binned in $2.5 \mathrm{~K} \lambda$ increments; insets show the region where disk emission is expected, with $5 \mathrm{~K} \lambda$ bins to increase signal to noise.

sample. The mean $M_{\text {disk }} / M_{\text {env }}$ is $6 \%$. Again we see no clear trend in the disk-to-envelope mass ratio with $T_{\text {bol }}$ within this young sample.

Our high detection rate in this complete sample suggests that disks must form very early in the star formation process. The Class 0 phase likely lasts approximately $0.2 \mathrm{Myr}$ (Enoch et al. 2009b), and we have detected disks in six of the nine Class 0 targets, which represent all Class 0 sources with $L_{\text {int }} \gtrsim 0.05 L_{\odot}$ and $M_{\mathrm{env}} \gtrsim 0.25 M_{\mathrm{env}}$ in the approximately $3.5 \mathrm{deg}$ cloud area. Thus, our results suggest that $2 / 3$ of embedded protostars in
Serpens have formed an $M_{\text {disk }}>0.02 M_{\odot}$ disk within the first $0.2 \mathrm{Myr}$.

Although there is no overlap between the two samples, the disk masses for our complete Serpens sample are roughly consistent with the Jørgensen et al. (2009) Class 0 disk masses. We also see similar disk to envelope mass ratios in Class 0 , ranging from $1 \%$ to $10 \%$ in both samples.

Jørgensen et al. (2009) found average disk masses in both Class 0 and Class I of $M_{\text {disk }}=0.05 M_{\odot}$, while Andrews $\&$ Williams (2007) found a lower average Class I disk mass 

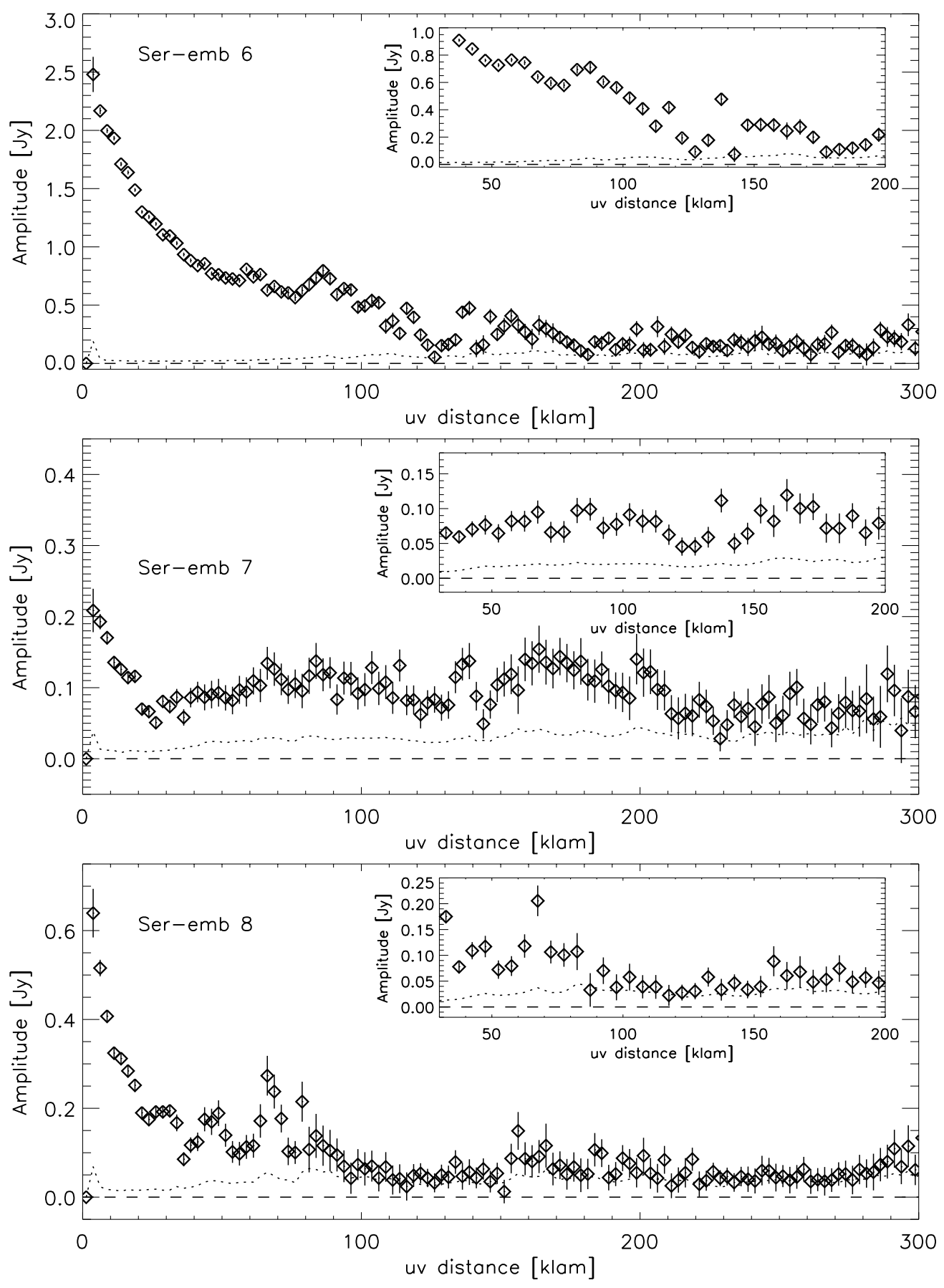

(b)

Figure 4. (Continued)

in Ophiuchus $\left(0.015 M_{\odot}\right)$. Our mean Class 0 disk mass is somewhat higher, $0.2 M_{\odot}$, but this may be a result of temperature variations with luminosity (e.g., Jørgensen et al. 2009) or the fact that we have not corrected for the envelope contribution at $50 \mathrm{~K} \lambda$. Given the uncertainties, our results appear to be consistent with the conclusion of Jørgensen et al. (2009) that disk masses are fairly constant through the embedded phases of protostellar evolution.

Such a scenario is supported by recent hydrodynamics simulations by Vorobyov (2009), which find time-averaged disk masses in Class 0 and Class I of $\sim 0.1 M_{\odot}$ for both viscous and self-gravitational disks. More robust measurements of disk masses and similar studies of the Class 0 populations in other clouds are needed to confirm these results.

\section{INDIVIDUAL SOURCES}

\subsection{Ser-emb 1}

Ser-emb 1 is located in Serpens Cluster B (Figure 1) and is associated with the single dish Bolocam $1.1 \mathrm{~mm}$ core Ser-Bolo 15 (Enoch et al. 2007). Ser-emb 1 has the lowest bolometric temperature ( $39 \mathrm{~K})$, and thus presumably is the least evolved, of all the protostars in Serpens. It is relatively isolated; a single Spitzer mid-infrared source is associated with the approximately 

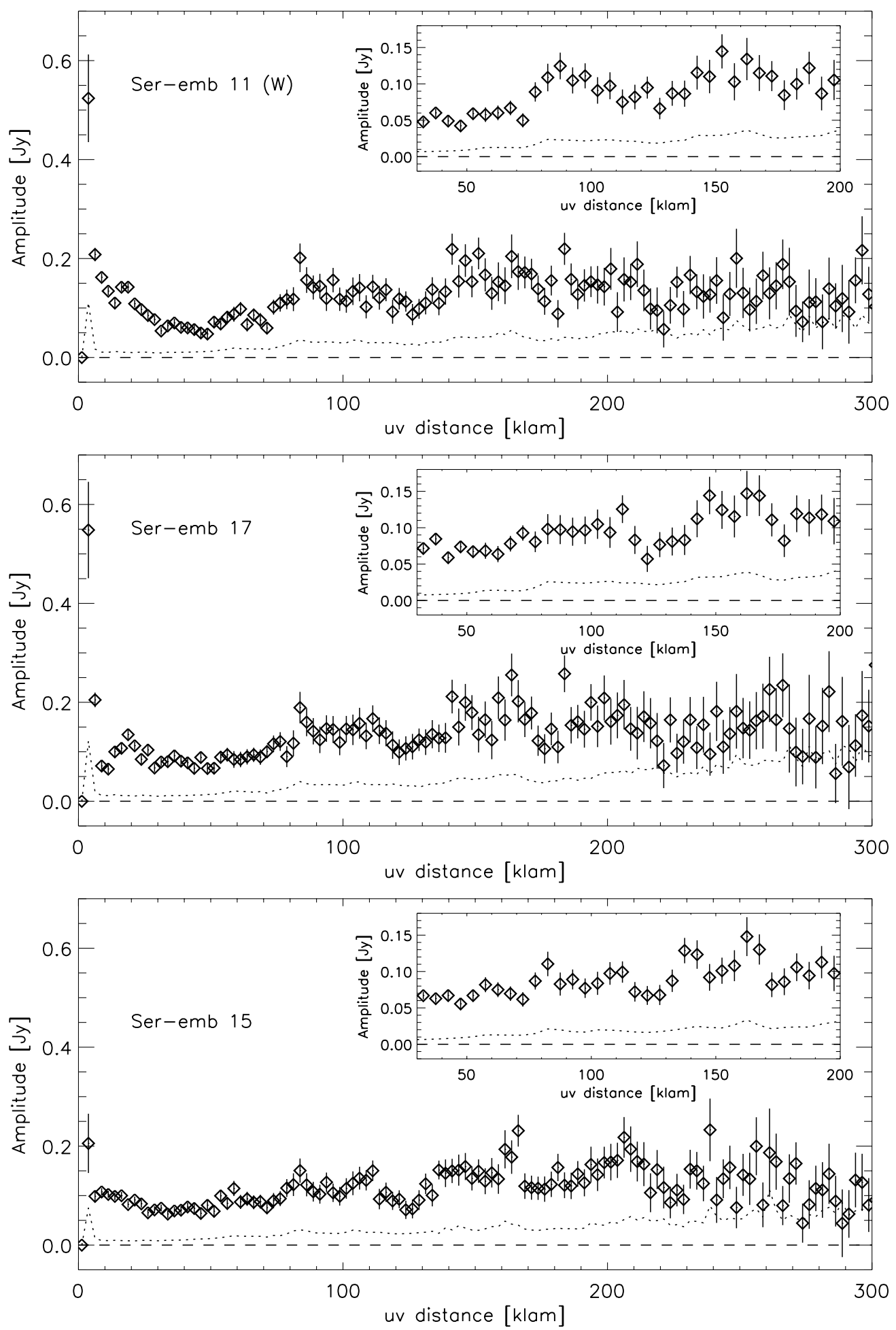

(c)

Figure 4. (Continued)

$10^{4} \mathrm{AU}$ diameter Bolocam core, and the nearest protostar is $\sim 70^{\prime \prime}\left(3 \times 10^{4} \mathrm{AU}\right)$ away.

The $230 \mathrm{GHz}$ map of Ser-emb 1 (Figure 3) shows a single compact source, which is centered on the mid-infrared source. The total flux from a Gaussian fit is $215 \mathrm{mJy}$ (Table 2), but the peak in the visibility plot (Figure 4, right) indicates a total flux in the CARMA data of $560 \mathrm{mJy}$. After scaling the $270 \mathrm{GHz}$ $(\lambda=1.1 \mathrm{~mm})$ Bolocam flux to $230 \mathrm{GHz}$, assuming a dust spectral index of $\beta=1.5$, we find that CARMA recovers approximately $95 \%$ of the single dish flux.

The visibility plot indicates substantial flux at intermediate $u v$-distances (30-100 K $\lambda$ ), which could be explained by either a resolved disk or a very compact envelope. The disk mass estimated from the flux at $50 \mathrm{~K} \lambda$ is $0.28 M_{\odot}$, or $9 \%$ of the total circum-protostellar mass, although this may be severely underestimated if the disk is partially resolved. 
Table 3

Estimated Disk Masses

\begin{tabular}{lcc}
\hline \hline ID & $\begin{array}{c}\text { Flux }(50 \mathrm{~K} \lambda) \\
(\mathrm{mJy})\end{array}$ & $\begin{array}{c}\text { Estimated } M_{\text {disk }} \\
\left(M_{\odot}\right)\end{array}$ \\
\hline Ser-emb 1 & 120 & 0.28 \\
Ser-emb 2 & $\ldots$ & $<0.02$ \\
Ser-emb 3 & $\ldots$ & $<0.02$ \\
Ser-emb 4 (N) & 26 & 0.05 \\
Ser-emb 5 & 17 & 0.04 \\
Ser-emb 6 & 750 & 1.7 \\
Ser-emb 7 & 66 & 0.15 \\
Ser-emb 8 & 110 & 0.25 \\
Ser-emb 9 & $\ldots$ & $<0.02$ \\
Ser-emb 11 (W) & 52 & 0.13 \\
Ser-emb 15 & 64 & 0.15 \\
Ser-emb 17 & 69 & 0.15 \\
\hline
\end{tabular}

Notes. The flux at $50 \mathrm{~K} \lambda$ is the average flux for $u v$-distances 40-60 K $\lambda$. Disk masses are estimated from Equation (1), and upper limits for Ser-emb 2, emb 3, and emb 9 are based on preliminary $110 \mathrm{GHz}$ maps (see the Appendix). Uncertainties in disk masses are at least $50 \%$, as discussed in the text.

\subsection{Ser-emb 4}

Ser-emb 4 is located in the Serpens Main cluster, in a crowded region (several other protostars within $1^{\prime}$ ). It is associated with the Bolocam core Ser-Bolo 28, as are Ser-emb 19 and Ser-emb 29. Most of the single dish flux, and thus most of the envelope mass, is likely associated with Ser-emb 4, which has a much lower $T_{\text {bol }}$ (54 K compared to $129 \mathrm{~K}$ and $374 \mathrm{~K}$, respectively).

The $230 \mathrm{GHz}$ map reveals three distinct sources; the northernmost is associated with the Ser-emb 4 mid-infrared source, while the other two appear to have no counterpart at $\lambda<70 \mu \mathrm{m}$. The eastern and southern sources are located 10" (4200 AU) and $15^{\prime \prime}$ (6200 AU) from the primary northern source, respectively.

Ser-emb $4(\mathrm{~N})$ has a diffuse extended envelope that is partially resolved out by the small synthesized beam. The extended nature of the envelope is also apparent in the narrow peak in the visibilities at small $u v$-distances. The peak visibility amplitude is $250 \mathrm{mJy}$, only $19 \%$ of the total single dish flux. This difference is accounted for by the fact that the Bolocam core breaks into several sources with the higher resolution of CARMA. A constant amplitude of $\sim 25 \mathrm{mJy}$ is seen out to $u v$ distance $\sim 100 \mathrm{~K} \lambda$, suggesting an unresolved disk component with $M_{\text {disk }} \sim 0.05 M_{\odot}$, approximately $1 \%$ of the total circumprotostellar mass. The two additional sources in the CARMA map also likely contribute to the single dish flux, making this $1 \%$ fraction a lower limit.

\subsection{Ser-emb 5}

Ser-emb 5 is located south of Cluster B and is one of the few embedded protostars not in one of the two main clusters. It is associated with the extended Bolocam core Ser-Bolo 7, as is Ser-emb 25, but the two sources are separated by $>1^{\prime}$. Ser-emb 5 has a low bolometric luminosity $\left(L_{\text {bol }}=0.4 L_{\odot}\right)$ and low envelope mass $\left(M_{\mathrm{env}}=0.6 M_{\odot}\right)$. It is the only Class 0 source in Serpens that is considered a Very Low Luminosity Object (VeLLO; see Dunham et al. 2008). VeLLOs are believed to be either very low mass protostars (i.e., proto-brown dwarfs) or stellar mass protostars with very low mass accretion rates (Dunham et al. 2008).

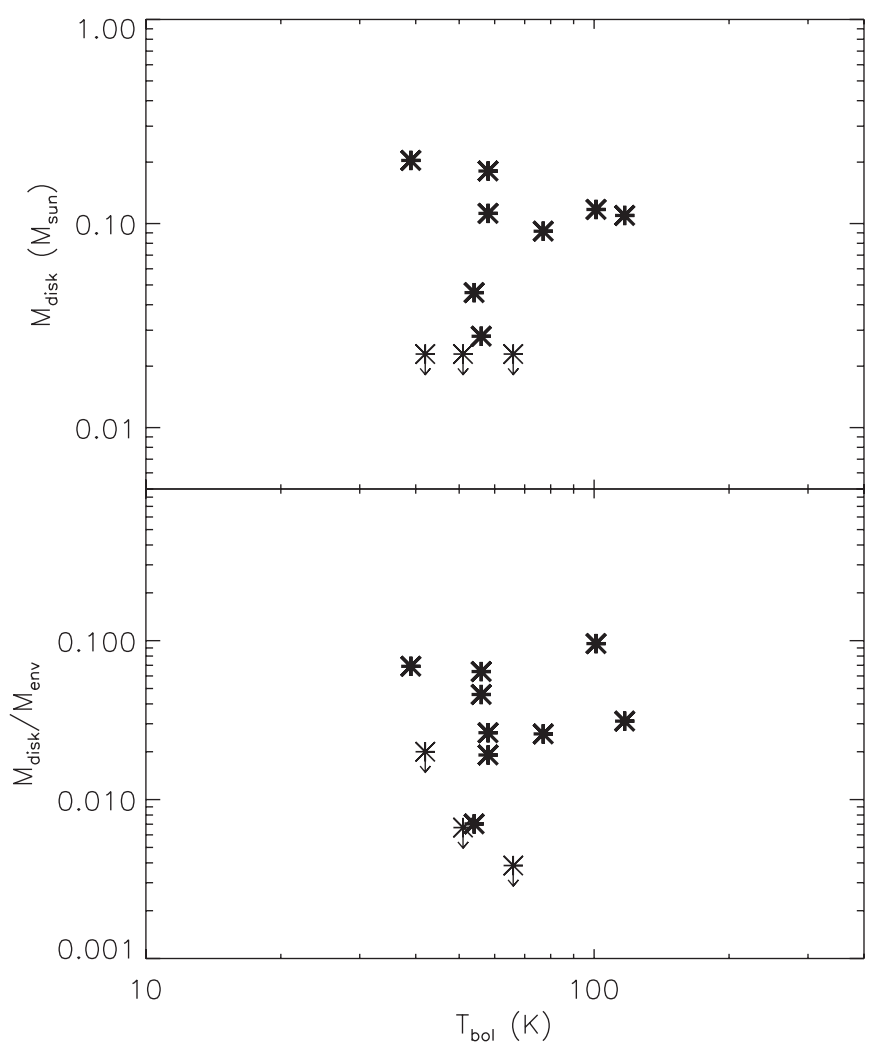

Figure 5. Estimated disk masses (top) and ratio of the estimated disk mass to envelope mass (bottom), as a function of bolometric temperature. Upper limits for three Class 0 sources in our sample are based on preliminary $110 \mathrm{GHz}$ maps (see the Appendix). No strong trend with $T_{\text {bol }}$ is seen for either the disk mass or disk to envelope mass ratio.

The $230 \mathrm{GHz}$ map contains a single, relatively compact source. The peak visibility amplitude is $68 \mathrm{mJy}$, accounting for $60 \%$ of the single dish flux. The flux at $50 \mathrm{~K} \lambda$ suggests a disk mass of $0.04 M_{\odot}$ or $6 \%$ of the total circum-protostellar mass. Ser-emb 5 has the lowest disk mass of our nine $230 \mathrm{GHz}$ targets.

\subsection{Ser-emb 6}

Ser-emb 6 is a well-known Class 0 protostar also known as Serpens FIRS 1 (Harvey et al. 1984) and Serpens SMM 1 (Casali et al. 1993). It is located in the Serpens Main cluster and is associated with the Bolocam core Ser-Bolo 23. Ser-emb 6 is the brightest Class 0 protostar in Serpens $\left(L_{\text {bol }}=28 L_{\odot}\right)$ and has the largest envelope mass $\left(M_{\mathrm{env}}=20 M_{\odot}\right)$ of any embedded protostar in the region.

The $230 \mathrm{GHz}$ map of Ser-emb 6 shows a complex source with both compact and extended emission. Approximately $58 \%$ of the single dish flux is recovered by CARMA, with a peak visibility amplitude of $2.3 \mathrm{Jy}$. In Paper I, we compared the CARMA visibilities, Spitzer IRS spectra, and broadband SED to a grid of detailed radiative transfer models calculated with RADMC (Dullemond \& Dominik 2004). The visibilities were best fit by a massive disk $\left(M_{\text {disk }}=2.5 M_{\odot}\right.$ when scaled for $\left.d=415 \mathrm{pc}\right)$, making up $13 \%$ of the total circumstellar mass and accounting for most of the flux at $u v$-distances $30-150 \mathrm{~K} \lambda$. Using only the flux at $50 \mathrm{~K} \lambda$ results in a disk mass of $1.7 M_{\odot}$ or $9 \%$ of the circumstellar mass, an underestimate of $30 \%$ compared to detailed modeling.

Choi (2009) suggests that Ser-emb 6 is a proto-binary source with a separation of $800 \mathrm{AU}$. This second source corresponds 
to the small peak seen to the northwest of Ser-emb 6 (Figure 3). Given its location along the outflow axis, and the clumpy nature of the envelope, we concluded in Paper I that this emission is associated with the outflow, but Choi (2009) argues that the source is a second disk component based on the spectral index from $3.5 \mathrm{~cm}$ to $7 \mathrm{~mm}$.

\subsection{Ser-emb 7}

Ser-emb 7 is located in Cluster B and is associated with the Ser-Bolo 8 core. Two other nearby protostars, Ser-emb $3\left(30^{\prime \prime}\right.$ away) and Ser-emb 9 (26" away), are associated with the same single dish core, but both were only marginally detected in initial $110 \mathrm{GHz}$ CARMA map (see the Appendix), suggesting that most of the core mass is associated with Ser-emb 7. Another possibility is that Ser-emb 3 and emb 9 have envelopes with a flat central density profile, which is resolved out by the interferometer. Neither Ser-emb 3 or emb 9 are included in our $230 \mathrm{GHz}$ study due to the lack of observed millimeter flux.

The $230 \mathrm{GHz}$ map of Ser-emb 7 shows a single extended source that appears elongated in the north-south direction. Only $29 \%$ of the single dish flux is recovered by CARMA. The flux at $50 \mathrm{~K} \lambda$ suggests a disk mass of $0.15 M_{\odot}$ or $4 \%$ of the total circum-protostellar mass, but the two nearby protostars may contribute to the single dish flux if they have envelopes that are resolved out by the interferometer.

\subsection{Ser-emb 8}

Ser-emb 8, also known as S68N (McMullin et al. 1994) or SMM 9 (Casali et al. 1993), is located in the Serpens Main cluster. Although two Class I protostars are located within the FWHM of the Bolocam core Ser-Bolo 22, the core is centered on Ser-emb 8 and the majority of envelope mass appears to be associated with this object.

The $230 \mathrm{GHz}$ map indicates a compact source at the position of Ser-emb 8, with two additional sources $\sim 12^{\prime \prime}$ and $\sim 20^{\prime \prime}$ away. Approximately $20 \%$ of the single dish flux is recovered with CARMA; as with Ser-emb 4 and emb 7, nearby sources were likely blended in the Bolocam beam. The flux at $50 \mathrm{~K} \lambda$ suggests a disk mass of $0.25 M_{\odot}$, or $3 \%$ of the total circumstellar mass, likely a lower limit as nearby sources are contributing to the single dish flux.

\subsection{Ser-emb 11 and Ser-emb 17}

Ser-emb 11 and emb 17 are both Class I sources associated with the Ser-Bolo 14 core, located in Cluster B. The two protostars are separated by $12^{\prime \prime}$ or $5000 \mathrm{AU}$, suggesting that they may be physically associated. They have similar SEDs, but Ser-emb 17 is brighter at IRAC wavelengths, resulting in a higher $T_{\text {bol }}$ (117 K compared to $77 \mathrm{~K}$ ). In Enoch et al. (2009b), the total $1.1 \mathrm{~mm}$ flux of Ser-Bolo 14 was divided evenly between them to determine the envelope masses.

The $230 \mathrm{GHz}$ map reveals two compact sources at the positions of Ser-emb 11 and emb 17. Ser-emb 11 is further resolved into two components separated by 2 ". 1 or $870 \mathrm{AU}$, with a flux ratio of approximately 2 . This is the only source in our sample with clear evidence for a binary or multiple component for separations $<2000 \mathrm{AU}$.

The visibility amplitudes of both Ser-emb 11 and emb 17 have narrow peaks, indicating very extended envelopes, and nearly constant amplitudes to $u v$-distance $>100 \mathrm{~K} \lambda$, suggesting compact disk components. Together, the peak visibility amplitudes of Ser-emb 11 and emb 17 account for approximately $60 \%$ of the total single dish flux. The fluxes at $50 \mathrm{~K} \lambda$ are similar, resulting in disk masses of $0.13 M_{\odot}$ (emb 11) and $0.15 M_{\odot}$ (emb 17), together approximately $4 \%$ of the total circumstellar mass. Note that visibilities and disk mass estimates are calculated only for the brighter emb 11 component $(\mathrm{W})$.

\subsection{Ser-emb 15}

Ser-emb 15 is a Class I source located to the east of Cluster $\mathrm{B}$, in the Ser-Bolo 24 core. The Bolocam core is also associated with the Class 0 source Ser-emb 2, which is $28^{\prime \prime}$ or 12000 AU away from emb 15 . Ser-emb 2 was not detected in preliminary $110 \mathrm{GHz}$ CARMA imaging (Figure 6). As there is significant $1.1 \mathrm{~mm}$ emission associated with emb 2 (see also Figure 2), the non-detection may be due to the lack of a compact disk combined with a flattened envelope density profile.

The $230 \mathrm{GHz}$ map of Ser-emb 15 shows a single compact source. The visibility amplitudes are nearly constant for $u v$ distances 5-200 $\mathrm{K} \lambda$, without the peak at small $u v$ distances expected for an extended envelope. Rather, the visibilities are consistent with a bright unresolved disk. The CARMA observations recover $54 \%$ of the total single dish flux associated with Ser-emb 15 . The flux at $50 \mathrm{~K} \lambda$ suggests a disk mass of $0.15 M_{\odot}$, only $13 \%$ of the total circum-protostellar mass. If a large fraction of the $1 \mathrm{~mm}$ flux is coming from the disk, however, the envelope mass calculated assuming $T_{D}=15 \mathrm{~K}$ is likely overestimated. Thus, Ser-emb 15 appears to have a compact disk surrounded by a relatively low-mass envelope, as expected if Class I sources have exhausted at least half of their envelope mass (e.g., André 1994).

\section{MULTIPLICITY}

The very high resolution millimeter maps presented here are also sensitive to binary and multiple sources, assuming that each source has a separate disk or envelope component. The multiplicity fraction at very early times is essential for understanding the formation of multiple systems, but very little is currently known about multiplicity in the Class 0 stage.

Although our observations do not have uniform sensitivity, the sensitivity to small scales (down to $250 \mathrm{AU}$ ) makes this sample a valuable addition to multiplicity studies. In only one source, Ser-emb 11, do we find strong evidence for multiplicity on scales $<2000 \mathrm{AU}\left(5^{\prime \prime}\right)$. Ser-emb 11 (E) and (W) are separated by $870 \mathrm{AU}$. Although Ser-emb 11 is a Class I source, it is very near the Class 0 cutoff $\left(T_{\text {bol }}=77 \mathrm{~K}\right)$, and is probably young enough to be included with the Class 0 sample. For our sample of nine sources, this yields a multiplicity ratio of approximately $10 \%$. Ser-emb 6 may also be a binary source with 800 AU separation based on the Very Large Array (VLA) observations of Choi (2009), so it is possible that the fraction is as high as $20 \%$.

Approximately $21 \%$ of Class I protostars have companions in the 250-2000 AU range (Connelley et al. 2008), with similar binary frequencies in more evolved Class II ( $\sim 16 \%$; Kraus et al. $2011)$ and main-sequence solar-type stars ( $\sim 10 \%$; Raghavan et al. 2010). Maury et al. (2010) recently observed five Class 0 protostars with subarcsecond resolution with IRAM-PdBI to study the fraction of binary and multiple systems down to $50 \mathrm{AU}$ separations. No Class 0 binaries were found with separations $<1500 \mathrm{AU}$, in contrast to the $\sim 20 \%$ multiplicity fractions observed in more evolved objects.

Combining our sample with the Maury et al. (2010) study, the resulting low Class 0 multiplicity fraction $(\sim 10 \%)$ is generally 
consistent with the suggestion of Maury et al. (2010) that the binary fraction in the 75-2000 AU range increases from the Class 0 to Class I stage, possibly due to dynamical evolution of initially very tight ( $<75 \mathrm{AU})$ or very wide ( $>2000 \mathrm{AU})$ systems. With still relatively few sources, however, the statistical uncertainties are too large to conclusively determine that Class 0 sources have fewer intermediate scale binaries than more evolved protostars.

\section{SUMMARY}

We have completed high-resolution, high-fidelity millimeter imaging of nine embedded protostars in the Serpens molecular cloud with the CARMA interferometer. Our sample is based on the complete $\left(M_{\text {env }} \gtrsim 0.25 M_{\odot}, L_{\text {bol }} \gtrsim 0.05 L_{\odot}\right)$ census of embedded protostars in Serpens from Enoch et al. (2009b) and includes six of the nine Class 0 sources in Serpens.

$230 \mathrm{GHz}$ continuum observations were completed in B, $\mathrm{C}, \mathrm{D}$, and $\mathrm{E}$ configurations, with small seven-point mosaics in the compact $\mathrm{D}$ and $\mathrm{E}$ configurations; the resulting images are sensitive to spatial scales from $10^{2}$ to $10^{4}$ AU. Our highresolution maps are also sensitive to binaries with separations $>250 \mathrm{AU}$. In only one source is there clear evidence for multiple components within $2000 \mathrm{AU}$, suggesting a tentative multiplicity fraction of only $10 \%$ for very deeply embedded protostars.

For unblended sources, our CARMA observations recover $50 \%-95 \%$ of the single dish Bolocam flux, assuming a dust spectral index of $\beta=1.5$. In all nine observed sources, $230 \mathrm{GHz}$ emission is seen on both large scales (a few $1000 \mathrm{AU}$ ), indicating an extended envelope, and small scales (down to $170 \mathrm{AU}$ ), indicating a compact disk component. Three of the nine Serpens Class 0 sources were not observed here due to non-detections or marginal detections in preliminary $110 \mathrm{GHz}$ CARMA data, and these sources likely do not have a significant disk. Thus, we see evidence for circum-protostellar disks in 6/9 Class 0 sources in Serpens.

We make a first estimate of the disk mass using the visibility amplitude at $50 \mathrm{~K} \lambda$, assuming an optically thin, unresolved disk with $T_{D}=30 \mathrm{~K}$ and a distance to Serpens of $415 \mathrm{pc}$ (Dzib et al.
2010). Resulting disk mass estimates range from $0.04 M_{\odot}$ to $1.7 M_{\odot}$, with a mean disk mass of $0.2 M_{\odot}$ when the three nondetections are included. Errors in disk masses may be $\pm 50 \%$, depending on the envelope contribution at $50 \mathrm{~K} \lambda$, the size of the disk, and the presence of optically thick emission. For a distance to Serpens of $260 \mathrm{pc}$, as assumed in our previous work, masses would decrease by a factor of 2.5. Our mean Class 0 disk mass is similar to that of the larger, but less well defined, Class 0 and Class I sample of Jørgensen et al. (2009).

Our high disk detection rate in a complete sample suggests that circum-protostellar disks are quite common even in the youngest protostars, with approximately $2 / 3$ of embedded protostars in Serpens having detectable $\left(M_{\text {disk }} \gtrsim 0.02 M_{\odot}\right)$ disks by 0.2 Myr.

Future work comparing the high-resolution millimeter data, as well as spectral information, with radiative transfer models will provide a more robust determination of the disk masses.

The authors thank the referee for comments and suggestions that improved this manuscript. Support for this work was provided by NASA through the Spitzer Space Telescope Fellowship Program, through a contract issued by the Jet Propulsion Laboratory (JPL), California Institute of Technology, under a contract with NASA. D.P.M. was supported by NASA through Hubble Fellowship grant HST-HF-51259.01 Support for CARMA construction was derived from the states of California, Illinois, and Maryland, the Gordon and Betty Moore Foundation, the Kenneth T. and Eileen L. Norris Foundation, the Associates of the California Institute of Technology, and the National Science Foundation. Ongoing CARMA development and operations are supported by the National Science Foundation under a cooperative agreement, and by the CARMA partner universities.

\section{APPENDIX}

\section{PRELIMINARY $110 \mathrm{GHz}$ DATA}

Preliminary $v=110 \mathrm{GHz}(\lambda=2.7 \mathrm{~mm})$ observations of the Bolocam $1.1 \mathrm{~mm}$ cores Ser-Bolo 8 (associated with mid-infrared identified protostars Ser-emb 3, emb 7, and emb 9) and Ser-Bolo
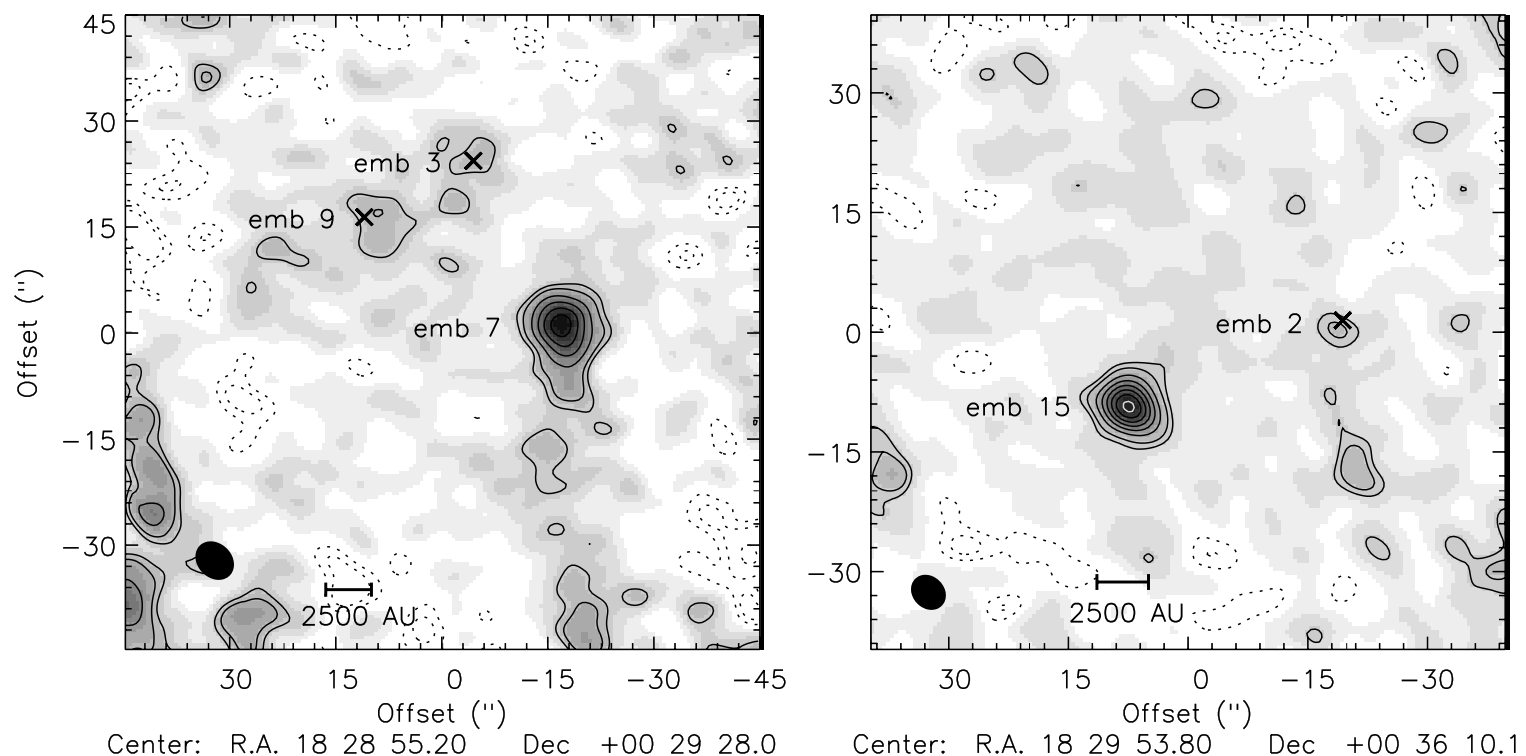

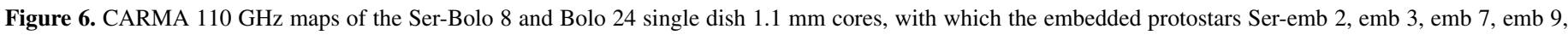

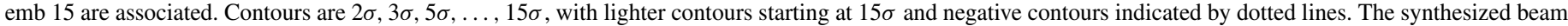

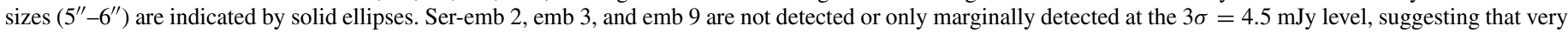
little of the mass traced by the single dish core is associated with these sources. 
24 (associated with Ser-emb 2 and emb 15) were completed with CARMA. The $6.1 \mathrm{~m}$ and $10.4 \mathrm{~m}$ antennas were used to obtain $110 \mathrm{GHz}$ continuum observations in the D and $\mathrm{E}$ antenna configurations between 2007 April 20 and 2007 September 4.

Three correlator bands were configured for continuum observations with $468.75 \mathrm{MHz}$ bandwidth, for a total bandwidth of $2.82 \mathrm{GHz}$. 1751+096 was observed approximately every 20 minutes for complex gain calibration. Absolute flux calibration was accomplished using 5 minute observations of MWC 349. The overall calibration uncertainty is approximately $\pm 20 \%$, from the reproducibility of the phase calibrator flux on nearby days. The passband calibrator, 1751+096, was observed for 15 minutes during each set of observations, and either optical (Corder et al. 2010) or radio pointing was performed every one to three hours. Data reduction was completed as described for the $230 \mathrm{GHz}$ data in Section 3.

The resulting $110 \mathrm{GHz}$ maps are shown in Figure 6. The rms noise in both maps is $\sim 1.5 \mathrm{mJy}$ and the synthesized beam sizes are $5^{\prime \prime}-6^{\prime \prime}$. Ser-emb 7 and emb 15 are both strongly detected. Ser-emb 3 and emb 9 are undetected at the $3 \sigma=4.5 \mathrm{mJy}$ level, although there is some $2 \sigma$ emission at the position of both sources. Ser-emb 2 is marginally detected at the $3 \sigma$ level.

Any detected flux in the compact D and E configurations may well include envelope emission, so we adopt an upper limit of $4.5 \mathrm{mJy}$ for the disk flux of all three sources. We convert this flux upper limit to a disk mass upper limit using Equation (1), where $\kappa_{2.7 \mathrm{~mm}}=0.003 \mathrm{~cm}^{2} \mathrm{~g}^{-1}$ is the dust opacity at $110 \mathrm{GHz}$. The opacity is extrapolated from Table 1 Column 6 of Ossenkopf \& Henning (1994) assuming $\kappa \propto v^{\beta}$ and $\beta=1.5$.

This yields a disk mass upper limit of $0.02 M_{\odot}$ for Ser-emb 2, emb 3, and emb 9. While the preliminary $110 \mathrm{GHz}$ maps are less sensitive than the subsequent $230 \mathrm{GHz}$ observations, non-detections place a fairly stringent upper limit on the total envelope mass, and thus on the disk mass.

\section{REFERENCES}

André, P. 1994, in The Cold Universe, ed. T. Montmerle et al. (Gif-sur-Yvette: Editions Frontieres), 179

Andrews, S. M., \& Williams, J. P. 2007, ApJ, 671, 1800

Brown, D. W., Chandler, C. J., Carlstrom, J. E., Hills, R. E., Lay, O. P., Matthews, B. C., Richer, J. S., \& Wilson, C. D. 2000, MNRAS, 319, 154

Casali, M. M., Eiroa, C., \& Duncan, W. D. 1993, A\&A, 275, 195

Chandler, C. J., Koerner, D. W., Sargent, A. I., \& Wood, D. O. S. 1995, ApJ, 449, 139

Chen, H., Myers, P. C., Ladd, E. F., \& Wood, D. O. S. 1995, ApJ, 445, 377

Choi, M. 2009, ApJ, 705, 1730

Connelley, M. S., Reipurth, B., \& Tokunaga, A. T. 2008, AJ, 135, 2526

Corder, S. A., Wright, M. C. H., \& Carpenter, J. M. 2010, Proc. SPIE, 7733 , 115

Davis, C. J., Matthews, H. E., Ray, T. P., Dent, W. R. F., \& Richer, J. S. 1999, MNRAS, 309, 141

Djupvik, A. A., André, Ph., Bontemps, S., Motte, F., Olofsson, G., Gålfalk, M., \& Florén, H.-G. 2006, A\&A, 485, 789
Dullemond, C. P., \& Dominik, C. 2004, A\&A, 417, 159

Dunham, M. M., Crapsi, A., Evans, N. J., II, Bourke, T. L., Huard, T. L., Myers, P. C., \& Kauffmann, J. 2008, ApJS, 179, 249

Dzib, S., Loinard, L., Mioduszewski, A. J., Boden, A. F., Rodrguez, L. F., \& Torres, R. M. 2010, ApJ, 718, 610

Eiroa, C., \& Casali, M. M. 1992, A\&A, 262, 468

Eisner, J. A., Hillenbrand, L. A., Carpenter, J. M., \& Wolf, S. 2005, ApJ, 635, 396

Enoch, M. L., Corder, S., Dunham, M. M., \& Duchêne, G. 2009a, ApJ, 707, 103

Enoch, M. L., Evans, N. J., II, Sargent, A. I., \& Glenn, J. 2009b, ApJ, 692, 973

Enoch, M. L., Glenn, J., Evans, N. J., II, Sargent, A. I., Young, K. E., \& Huard, T. L. 2007, ApJ, 666, 982

Evans, N. J., II, Allen, L. E., Blake, G. A., Boogert, A. C. A., Bourke, T., Harvey, P. M., \& Kessler, J. E., et al. 2003, PASP, 115, 965

Evans, N. J., II., et al. 2009, ApJS, 181, 321

Galli, D., \& Shu, F. H. 1993, ApJ, 417, 220

Glenn, J., et al. 2003, Proc. SPIE, 4855, 30

Harvey, D. W. A., Wilner, D. J., Lada, C. J., Myers, P. C., \& Alves, J. F. 2003, ApJ, 596, 383

Harvey, P. M., Wilking, B. A., \& Joy, M. 1984, ApJ, 278, 156

Harvey, P. M., et al. 2006, ApJ, 644, 307

Harvey, P. M., et al. 2007, ApJ, 663, 1139

Hatchell, J., Fuller, G. A., Richer, J. S., Harries, T. J., \& Ladd, E. F. 2007, A\&A, 468, 1009

Hogerheijde, M. R., van Dishoeck, E. F., \& Salverda, J. M. 1999, ApJ, 513, 350

Hurt, R. L., \& Barsony, M. 1996, ApJ, 460, L45

Jørgensen, J. K., Bourke, T. L., Myers, P. C., Schöier, F. L., van Dishoeck, E. F., \& Wilner, D. J. 2005a, ApJ, 632, 973

Jørgensen, J. K., Johnstone, D., Kirk, H., \& Myers, P. C. 2007, ApJ, 656, 293

Jørgensen, J. K., Johnstone, D., Kirk, H., Myers, P. C., Allen, L. E., \& Shirley, Y. L. 2008, ApJ, 683, 822

Jørgensen, J. K., van Dishoeck, E. F., Visser, R., Bourke, T. L., Wilner, D. J., Lommen, D., Hogerheijde, M. R., \& Myers, P. C. 2009, A\&A, 507, 861

Jørgensen, J. K., et al. 2005b, ApJ, 631, L77

Keene, J., \& Masson, C. R. 1990, ApJ, 355, 635

Kraus, A. L., Ireland, M. J., Marinache, F., \& Hillenbrand, L. A. 2011, ApJ, 731,8

Larsson, B., et al. 2000, A\&A, 363, 253

Looney, L. W., Mundy, L. G., \& Welch, W. J. 2000, ApJ, 529, 477

Looney, L. W., Mundy, L. G., \& Welch, W. J. 2003, ApJ, 592, 255

Looney, L. W., Tobin, J. J., \& Kwon, W. 2007, ApJ, 670, L131

Maury, A. J., et al. 2010, A\&A, 512, 40

McMullin, J. P., Mundy, L. G., Wilking, B. A., Hezel, T., \& Blake, G. A. 1994, ApJ, 424, 222

Myers, P. C., \& Ladd, E. F. 1993, ApJ, 413, 47

Ossenkopf, V., \& Henning, Th. 1994, A\&A, 291, 943

Pérez, L. M., et al. 2010, ApJ, 724, 493

Raghavan, D., et al. 2010, ApJS, 190, 1

Robitaille, T. P., Whitney, B. A., Indebetouw, R., Wood, K., \& Denzmore, P. 2006, ApJS, 167, 256

Sault, R. J., Teuben, P. J., \& Wright, M. C. H. 1995, in ASP Conf. Ser. 77, Astronomical Data Analysis Software and Systems IV, ed. R. A. Shaw, H. E. Payne, \& J. J. E. Hayes (San Francisco, CA: ASP), 433

Steer, D. G., Dewdney, P. E., \& Ito, M. R. 1984, A\&A, 137, 159

Terebey, S., Shu, F. H., \& Cassen, P. 1984, ApJ, 286, 529

Testi, L., \& Sargent, A. I. 1998, ApJ, 508, L94

Visser, R., van Dishoeck, E. F., Doty, S. D., \& Dullemond, C. P. 2009, A\&A 495,881

Vorobyov, E. I. 2009, ApJ, 692, 1609 